\title{
Potential Kick Velocity distribution of black hole X-ray binaries and implications for natal kicks
}

\author{
P. Atri, ${ }^{1 \star}$ J. C. A. Miller-Jones,${ }^{1}$ A. Bahramian, ${ }^{1}$ R. M. Plotkin, ${ }^{1}$ P. G. Jonker, ${ }^{2,3}$ \\ G. Nelemans, ${ }^{3,4}$ T. J. Maccarone, ${ }^{5}$ G. R. Sivakoff, ${ }^{6}$ A. T. Deller, ${ }^{7}$ S. Chaty ${ }^{8}$ \\ M. A. P. Torres, ${ }^{9,3}$ S. Horiuchi, ${ }^{10}$ J. McCallum, ${ }^{11}$ T. Natusch,,${ }^{12}$ C. J. Phillips,${ }^{13}$ \\ J. Stevens, ${ }^{13}$ S. Weston ${ }^{12,13}$
}

${ }^{1}$ International Centre for Radio Astronomy Research, Curtin University, GPO Box U1987, Perth, WA 6845, Australia

${ }^{2}$ SRON, Netherlands Institute for Space Research, Sorbonnelaan 2, NL-3584 CA Utrecht, the Netherlands

${ }^{3}$ Department of Astrophysics/IMAPP, Radboud University Nijmegen, POBox 9010, NL-6500 GL, Nijmegen, the Netherlands

${ }^{4}$ Institute of Astronomy, KU Leuven, Celestijnenlaan 200D, B-3001 Leuven, Belgium

${ }^{5}$ Department of Physics, Box 41051, Science Building, Texas Tech University, Lubbock, TX 79409-1051, USA

${ }^{6}$ Department of Physics, University of Alberta, CCIS 4-183, Edmonton, AB T6G 2E1, Canada

${ }^{7}$ Centre for Astrophysics and Supercomputing, Swinburne University of Technology, Mail Number H11, P.O. 218, Hawthorn, VIC 3122 Australia

${ }^{8}$ AIM, CEA, CNRS, Universitè Paris-Saclay, Universitè Paris Diderot, Sorbonne Paris Citè, F-91191 Gif-sur-Yvette, France

${ }^{9}$ Departamento de Astrofísica, Universidad de La Laguna, E-38206, Santa Cruz de Tenerife, Spain

${ }^{10}$ CSIRO Astronomy and Space Science, Canberra Deep Space Communication Complex, Tuggeranong, ACT 2901, Australia

${ }^{11}$ University of Tasmania, Private Bag 37, Hobart, Tasmania, 7001, Australia

${ }^{12}$ Institute for Radio Astronomy 85 Space Research, AUT University, 1010, Auckland, New Zealand

${ }^{13}$ CSIRO, P.O. Box 76, Epping, NSW 1710, Australia

Accepted XXX. Received YYY; in original form ZZZ

\begin{abstract}
We use Very Long Baseline Interferometry to measure the proper motions of three black hole X-ray binaries (BHXBs). Using these results together with data from the literature and Gaia-DR2 to collate the best available constraints on proper motion, parallax, distance and systemic radial velocity of $16 \mathrm{BHXBs,} \mathrm{we} \mathrm{determined} \mathrm{their} \mathrm{three}$ dimensional Galactocentric orbits. We extended this analysis to estimate the probability distribution for the potential kick velocity (PKV) a BHXB system could have received on formation. Constraining the kicks imparted to BHXBs provides insight into the birth mechanism of black holes (BHs). Kicks also have a significant effect on $\mathrm{BH}-\mathrm{BH}$ merger rates, merger sites, and binary evolution, and can be responsible for spin-orbit misalignment in BH binary systems. $75 \%$ of our systems have potential kicks $>70 \mathrm{~km} \mathrm{~s}^{-1}$. This suggests that strong kicks and hence spin-orbit misalignment might be common among BHXBs, in agreement with the observed quasi-periodic X-ray variability in their power density spectra. We used a Bayesian hierarchical methodology to analyse the PKV distribution of the BHXB population, and suggest that a unimodal Gaussian model with a mean of $107 \pm 16 \mathrm{~km} \mathrm{~s}^{-1}$ is a statistically favourable fit. Such relatively high PKVs would also reduce the number of BHs likely to be retained in globular clusters. We found no significant correlation between the $\mathrm{BH}$ mass and PKV, suggesting a lack of correlation between $\mathrm{BH}$ mass and the $\mathrm{BH}$ birth mechanism. Our Python code allows the estimation of the PKV for any system with sufficient observational constraints.
\end{abstract}

Key words: astrometry-proper motions-parallaxes-stars:black holestars:kinematics and dynamics-X-rays:binaries 
whereby the massive progenitor star collapses directly into a BH with almost no mass ejection (e.g. Reynolds et al. 2015; Adams et al. 2017), or (b) delayed formation in a supernova (SN), wherein matter ejected during the SN falls back onto the proto-neutron star, creating a BH (e.g. Gourgoulhon 1991; Woosley \& Weaver 1995; Brandt et al. 1995). The theoretical mass distribution derived by Fryer \& Kalogera (2001) suggests that beyond a critical progenitor mass of $40 M_{\odot}$ (assuming no mass loss through winds), BHs form via direct collapse and a supernova explosion does not occur. However, more recent work suggests that the formation of $\mathrm{BHs}$ by direct collapse (implosion) has a wider progenitor mass range, interspersed by narrow pockets of progenitor masses that undergo a supernova explosion to become a BH (Sukhbold et al. 2016). Obtaining observational evidence about how a $\mathrm{BH}$ was born for a range of $\mathrm{BH}$ masses could help in understanding the relation between the $\mathrm{BH}$ birth model and BH mass.

Isolated stellar mass BHs are difficult to observe. Thus, black hole X-ray binaries (BHXBs) can be used as probes to understand the birth mechanism of BHs in binary systems. If a $\mathrm{BH}$ in a binary system is formed due to a $\mathrm{SN}$ explosion, the sudden mass loss from the binary system will change the centre of mass of the remnant binary system. Even if the $\mathrm{SN}$ explosion is symmetric in the frame of reference of the progenitor, the change in the centre of mass of the binary system will give the remnant binary system a recoil kick. This kick is referred to as the Blaauw kick (Blaauw 1961). The ejected mass in the SN needs to be less than half the total mass of the system in order for the remnant binary system to stay bound. This corresponds to a maximum recoil kick of a few tens of $\mathrm{km} \mathrm{s}^{-1}$, depending on the binary mass, progenitor velocity, orbital period, and ejected mass of the system (Nelemans et al. 1999).

BHXBs that have peculiar velocities higher than the Blauuw kick the system could have acquired due to symmetric mass loss, need some other mechanism to explain the high velocities. The space velocity distribution of pulsars was shown to have a mean of $450 \mathrm{~km} \mathrm{~s}^{-1}$ with a highvelocity tail (Lyne \& Lorimer 1994). Verbunt et al. (2017, V17 hereafter) used accurately measured pulsar proper motions and parallaxes applying a Bayesian method to estimate distances from parallaxes. They found that the velocity distribution was wider than can be fit by a single Maxwellian, and has two prominent peaks, at $120 \mathrm{~km} \mathrm{~s}^{-1}$ and $540 \mathrm{~km} \mathrm{~s}^{-1}$. The high velocity kicks (Lyne \& Lorimer 1994) have been attributed to a variety of mechanisms during the SN explosion. Even if the matter ejected in the SN explosion is symmetric, the anisotropy in the neutrino emission caused by strong magnetic fields could accelerate pulsars to high velocities (Chugai 1984; Sagert \& Schaffner-Bielich 2008). Other mechanisms that could be responsible for high kick velocities include supersonic jet generation during a stellar core collapse (Khokhlov et al. 1999), or an intrinsically asymmetric explosion due to hydrodynamic perturbations in the SN core (Janka 2017).

Since these kick mechanisms occur prior to the fallback of matter on the proto-neutron star and consequent BH formation (Gourgoulhon \& Haensel 1993), it is plausible that $\mathrm{BHs}$ formed by the fallback process should also receive such strong natal kicks. It is expected that the velocity kicks BHs receive will be smaller than NS velocity kicks because BHs are more massive than NSs. On the other hand, fallback of slower moving ejecta from the SN explosion could accelerate the BH (Janka 2013). This would imply that BHs born in a $\mathrm{SN}$ explosion could also get large kicks at birth. BHs born by direct collapse are expected to receive kicks lower than those obtained due to birth in SN explosion (Belczynski et al. 2002). Hence, natal kick measurements provide effective probes to differentiate between the SN and direct collapse birth pathways.

In the absence of direct kick measurements, the height of known BHXBs above the Galactic plane has been used as a proxy (e.g. White \& van Paradijs 1996; Jonker \& Nelemans 2004) given that the majority of the progenitor systems are closely confined to the plane. Various natal kick distributions were used to simulate BHXB populations and it was found that natal kicks are essential to explain the large displacement of a number of systems from the Galactic plane (Repetto et al. 2012, 2017). However, Mandel (2016) showed that for extreme assumptions (i.e. all systems are currently at maximum z) BH kicks of $>100 \mathrm{~km} \mathrm{~s}^{-1}$ are not required to explain the observed distribution of heights $(|z|)$ above the Galactic plane. Measuring space velocities and positions of BHXBs provides extra information that helps to relate the $|z|$-distribution to the natal kicks received by the BHs and in turn resolve this discrepancy.

\section{$1.1 \quad$ BH-BH mergers}

With the discovery of $\mathrm{BH}$ mergers from gravitational wave (GW) events (Abbott et al. 2016a), we are achieving new insights into the stellar-mass BH mass distribution (The LIGO Scientific Collaboration \& The Virgo Collaboration 2018; Abbott et al. 2016b) and stellar mass BH formation. According to theoretical models, BH-BH binaries are formed due to dynamical interactions in high density clusters (Fabian et al. 1975; Goodman \& Hut 1993; Sigurdsson \& Hernquist 1993; Sigurdsson \& Phinney 1993; Benacquista \& Downing 2013) eg., in globular clusters (GCs) (Rodriguez et al. 2016) and Galactic nuclear clusters. They can also be formed due to hierarchical three body interactions (Antonini et al. 2018), or in the Galactic field, which hosts the astrophysical stellar binaries that evolve into BH-BH binaries (Belczynski et al. 2002; Postnov \& Yungelson 2014).

Strong natal kicks could lead to the BHs being kicked out of a GC before they could become BH-BH binaries, or result in unbinding the binary system. This would hinder $\mathrm{BH}-\mathrm{BH}$ binary formation and in turn reduce the rate of $\mathrm{BH}-$ $\mathrm{BH}$ mergers. Natal kicks are thus a deciding factor in the rate of BH-BH mergers (Wysocki et al. 2018). Natal kicks are also considered to be responsible for spreading the BH binary merger sites further away from the host halo (Kelley et al. 2010). While it is currently poorly constrained, the BH natal kick distribution is a key parameter in N-body simulations of GCs, used to estimate the number of BHs that are retained or ejected upon formation and due to subsequent dynamical interactions (e.g. Strader et al. 2012; Heggie \& Giersz 2014; Giesler et al. 2018). Thus, the natal kick distribution has important implications for the likelihood of finding $\mathrm{BH}-\mathrm{BH}$ binaries, and also BHXBs in GCs. 


\subsection{Existing natal kick constraints}

While we know of $\sim 60$ strong candidate $\mathrm{BH}$ systems (Tetarenko et al. 2016a; Corral-Santana et al. 2016), only seven BHXB candidates have been analysed to determine if they were born in a supernova with substantial mass ejection, rather than a direct collapse. System parameters like component masses, orbital period, donor effective temperature and the three dimensional motion of BHXBs need to be well constrained if the complete evolutionary history of these BHXB systems is to be mapped. The Galactocentric orbits of such systems with well known parameters could be integrated back to a reasonable range of their ages, thus the velocity of the system immediately after the $\mathrm{BH}$ birth could be determined. Such an analysis has been conducted for three systems, namely, XTE J1118+40 (Willems et al. 2005), GRO J1655-40 (Fragos et al. 2009) and Cyg X-1 (Wong et al. 2012).

The post SN peculiar velocity of XTE J1118+40 along with the assumption that the system was born in the Galactic plane indicated that the system received an asymmetric natal kick (Fragos et al. 2009). The high peculiar velocity could also mean that the system was a GC escapee (Mirabel et al. 2001; Gualandris et al. 2005), in which case the assumption that it was born in the Galactic plane would not hold. It was suggested that GRO J1655-40 was born in a SN and received a kick when the $\mathrm{BH}$ was formed (Brandt et al. 1995; Mirabel et al. 2002; Willems et al. 2005), though the strength of the kick did not require an asymmetric explosion (Nelemans et al. 1999). Cyg X-1 was found to have received a small kick velocity at birth (Mirabel \& Rodrigues 2003; Wong et al. 2012).

Only the peculiar velocities were used to infer whether the kicks BHXB systems received were high enough to suggest a SN origin for the other four systems (e.g. Mirabel et al. 2001; Mirabel \& Rodrigues 2003; Dhawan et al. 2007; MillerJones et al. 2009a; Gandhi et al. 2019). This has been done by measuring the proper motion of the BHXBs, and combining them with the line-of-sight velocities and the distances to the systems to measure the velocities of the systems with respect to the local standard of rest (i.e. the peculiar velocity). This gives the full three dimensional motion of the BHXBs, which enables us to trace the Galactocentric orbits through the Galactic potential. A low peculiar velocity was measured for GRS 1915+105 (Dhawan et al. 2007; Reid et al. 2014), which suggested that the system could have been born by direct collapse. The inferred peculiar velocities of V404 Cyg (Miller-Jones et al. 2009a), VLA J2130+12 (Tetarenko et al. 2016b) and MAXI J1836-194 (Russell et al. 2015) were more consistent with a symmetric SN explosion. This is a small sample size, with estimations of the minimum natal kick having been made using different methods. Thus in order to draw statistically robust conclusions about the natal kick distribution of BHs, we need to increase this sample size and adopt a more systematic approach. In this paper we increase the sample size of estimated kick velocities of BHXBs to 16, by combining measured proper motions, systemic radial velocities and distances to these systems.

\subsection{Peculiar velocity and Potential kick velocity}

Using the current peculiar velocity of a system to infer the strength of the kick the system might have received at birth has a few limitations. Peculiar velocity is the current three dimensional velocity of a system relative to the local standard of rest and thus changes based on the epoch of observation. Also, using peculiar velocity relative to that expected from Galactic rotation as an indicator of natal kick velocity could be misleading for sources that are far away from the Galactic plane. Our analysis tries to minimise these shortcomings by instead using the peculiar velocity of the system when it crosses the Galactic plane, which we refer to as the potential kick velocity (PKV).

In this paper we have combined the capabilities of VLBI and Gaia to get observationally constrained $\mathrm{BH}$ potential kick velocity probability distributions for 16 systems. In Section 2 we identify the BHXB sample we used for our analysis and mention the observational biases this sample suffers from. In Section 3 we discuss the data acquisition and reduction procedures followed to measure proper motions for GX 339-4, GRS 1716-249 and Swift J1753.5-0127, which is followed by Section 4 where we present the proper motion measurements of the above mentioned three systems. In Section 5 we go through the details of distance estimation from parallax measurements using a Milky Way prior (Section 5.1) and the systemic radial velocity estimates for the systems that did not have measured systemic radial velocity in literature (Section 5.2). Section 6 explains the Monte Carlo (MC) code we developed to determine the PKV probability distributions and present the potential kick velocity distributions for all 16 sources. Section 7 discusses the implications of these results.

\section{DATA}

\subsection{BHXB sample}

To determine the three dimensional motion of BHXBs, we need to combine the proper motion, systemic radial velocity and distances to these systems. The literature already contains estimates of the current peculiar velocity for seven of our systems, XTE J1118+480 (Mirabel et al. 2001), GRO 1655-40 (Mirabel et al. 2002), Cyg X-1 (Mirabel \& Rodrigues 2003), GRS 1915+105 (Dhawan et al. 2007), V404 Cyg (Miller-Jones et al. 2009a), MAXI J1836-194 (Russell et al. 2015) and VLA J2130+12 (Tetarenko et al. 2016b). Gaia in its second data release (DR2) measured the proper motions of 11 BHXBs, three of which were improvements on previous measurements (Cyg X-1, GRO J1655-40 and XTE J1118+480; Mirabel et al. 2001, 2002; Mirabel \& Rodrigues 2003). Gandhi et al. (2019) estimated the current peculiar velocities for BHXBs for which Gaia-DR2 (Gaia Collaboration et al. 2018) measured proper motions and parallaxes. We use these proper motion and parallax measurements along with the Very Long Baseline Interferometry (VLBI; Shapiro et al. 1979) measurements present in the literature (based on whichever was more precise) for our analysis.

Typical BHXBs have proper motions of a few mas $\mathrm{yr}^{-1}$, which can be measured by the Gaia satellite (Lindegren et al. 2016) or with VLBI. The quiescent optical brightness of 
BHXBs is usually near to or below the limiting magnitude of the Gaia satellite, and they only get bright enough for high precision astrometry with Gaia when they are in outburst. This makes proper motion measurements challenging for many of these sources. Gaia would also not be able to detect BHXBs towards the Galactic bulge due to high extinction. Thus, triggered VLBI observations when a BHXB goes into an outburst can probe systems not accessible to Gaia. We used VLBI to measure the proper motions of three new sources; GX 339-4, Swift J1753.5-0127 and GRS 1716-249.

\subsection{Sample biases}

Our sample set of 16 BHXB suffers from certain observational biases. There are a few systems in our sample that are not dynamically confirmed BHs but are BHXB candidates (see Tetarenko et al. (2016b) for a summary of all BHXB candidates). Notably, the nature of VLA J2130+12 that is a BHXB detected in quiescence (Kirsten et al. 2014) is still under debate (Tetarenko et al. 2016a). We include Cyg X-1 in our analysis even though it is a younger, high mass X-ray binary (HMXB) with a potentially more massive donor star than that of a typical low mass X-ray binary. This is because it has well constrained parameters and thus is a good test source.

We note that almost all BHXBs in our sample have been in outburst at some point, except VLA J2130+12. During outbursts typical BHXBs reach X-ray peak luminosities of $>10^{37-39} \mathrm{ergs} \mathrm{s}^{-1}$. Thus the sample is devoid of any very faint X-ray transients (Wijnands et al. 2006), which have peak luminosities of $10^{36-37} \mathrm{ergs} \mathrm{s}^{-1}$ (Heise 1999). We are biased against observing $\mathrm{BHs}$ that received kicks strong enough to unbind the binary system, as we can only observe BHs in Xray binaries. We may also be observationally biased against distant, low kick systems as they will be very close to the Galactic plane and therefore will be highly extincted.

\section{VLBI OBSERVATIONS AND DATA REDUCTION}

To measure the proper motions of GX 339-4, GRS 1716-249 and Swift J1753.5-0127, we used the Very Long Baseline Array (VLBA), the European VLBI Network (EVN) and the Australian Long Baseline Array (LBA). The observational setups are summarised in Table 1 . The hard X-ray spectral state of BHXBs is associated with the rising and decaying phases of the outbursts (Belloni \& Motta 2016). The radio jets during the hard spectral state are compact, steady and are causally connected to the BH (Fender et al. 2009). Thus the hard state is an ideal phase during the outburst to conduct astrometry. High precision astrometry can be conducted even if the target image is resolved (Reid et al. 2011). The observations need to be separated in time by at least a couple of months to provide a sufficient time baseline for a proper motion measurement. Thus, depending on how long the source stays in a hard state, we need to observe it over one or multiple outbursts.

The data were correlated using the DiFX software correlator (Deller et al. 2011) and reduced using the Astronomical Image Processing System (AIPS 31DEC17; Greisen 2003). Observations of bright fringe finder sources were used to correct for non-zero instrumental delays and rates. The observations of the target were bracketed by shorter observations of a phase reference calibrator, located as close to the target as possible (preferably $<2^{\circ}$; Pradel et al. 2006). The details of the calibrators used for each target are summarised in Table 2.

For observations made at frequencies higher than $5 \mathrm{GHz}$ using the VLBA, numerous widely separated calibrators were observed in quick succession (geodetic blocks) for $\sim 30$ minutes at the start and end of each observing run. Geodetic blocks help in determining any error in the estimated zenith tropospheric delay and thus improve astrometric accuracy. The multi-band delays were corrected using standard AIPS procedures (AIPS Memo 110). The overheads associated with observing a geodetic block with the LBA and the EVN were high due to the large dish sizes and consequent slow slew rates of the dishes involved, and hence we did not observe geodetic blocks with the LBA and the EVN. The stations included in the LBA and the EVN are not solely for the purpose of VLBI, and so have infrequent formal VLBI observing sessions. For any transients that have to be observed out of the standard observing sessions, the source is observed with whatever stations are available. This gives varying sensitivity and resolution from epoch to epoch.

\subsection{GX 339-4}

GX 339-4 is a low mass black hole X-ray binary (LMXB), which was first detected in 1973 (Markert et al. 1973). This system goes into frequent outbursts, with 20 outbursts since its detection (Tetarenko et al. 2016a). Hence, astrometry over multiple outbursts is possible. The optical emission from the accretion disc of the system even during the low luminosity quiescent phase makes the detection of the donor star difficult (Shahbaz et al. 2001). Recently, NIR spectra of GX 339-4 were obtained using the VLT/X-Shooter during quiescence, which led to a systemic radial velocity measurement of $\gamma=26 \pm 2 \mathrm{~km} \mathrm{~s}^{-1}$ (Heida et al. 2017) and a distance estimate of $9 \pm 4 \mathrm{kpc}$ for the system. GX 339-4 was observed on three epochs (Table 1) using the LBA during the hard states of three different outbursts (Homan \& Belloni 2005; Buxton et al. 2013; Yan et al. 2014) spanning over 4 years. We observed at a frequency of $8.4 \mathrm{GHz}$ in all three epochs for maximum sensitivity.

\subsection{GRS 1716-249}

GRS 1716-249 (Nova Oph 1993) was discovered (Ballet et al. 1993) as an X-ray transient in Ophiuchus in 1993. della Valle et al. (1994) conducted a photometric and spectroscopic analysis of GRS 1716-249 and concluded that the source is at a distance of $2.4 \pm 0.4 \mathrm{kpc}$. The mass of the primary in the system is $>4.9 \mathrm{M}_{\odot}$ (Masetti et al. 1996). The systemic radial velocity of this system is not yet known. After a prolonged quiescent period (Negoro et al. 2016) following some renewed activity in 1995 (Karitskaya \& Goranskij 1995), the source went into outburst again in December 2016, but did not reach a soft state. The source was observed at three epochs under the LBA program V447 (Table 1 and Table 2 ), and the observations were spaced a few months apart. The last two observations were taken right after the source 
Table 1. Summary of the VLBI observations. Station codes: Ak - ASKAP; At - phased up ATCA; Br - Brewster; Cd - Ceduna; Ef Effelsberg; Fd - Fort Davis; Gb - Green Bank; Hh - Hartebeesthoek; Hn - Hancock; Ho - Hobart; Jb - Jodrell Bank Mk II; Ke - Katherine; Kp - Kitt Peak; La - Los Alamos; Mc - Medicina; Mk - Mauna Kea; Mp - Mopra; Nl - North Liberty; Nt - Noto; On - Onsala; Ov Owens Valley ; Pa - Parkes; Pt - Pie Town; Sc - St. Croix; Td - the $34 \mathrm{~m}$ DSS36 antenna at Tidbinbilla; Ti - the $70 \mathrm{~m}$ DSS43 antenna at Tidbinbilla; Tr - Torun; Wa - Warkworth 30m (Woodburn et al. 2015); Wb - Westerbork; Ww - Warkworth 12m; Yg - Yarragadee; Ys Yebes

\begin{tabular}{|c|c|c|c|c|c|c|c|}
\hline Target & Array & Code & Date & (UTC) & $\begin{array}{l}\text { Freq. } \\
(\mathrm{GHz})\end{array}$ & $\begin{array}{c}\text { Time } \\
\text { on } \\
\text { Source } \\
\text { (mins) }\end{array}$ & Stations \\
\hline \multirow[t]{3}{*}{ GX 339-4 } & \multirow[t]{3}{*}{ LBA } & V430A & 2011 Apr 03 & 55654.84 & 8.4 & 368.2 & At, Cd, Ho, Mp, Pa, Ti \\
\hline & & V486B & 2013 Aug 15 & 56520.20 & 8.4 & 193.9 & Ak, At, Cd, Hh, Ho, Ke, Mp, Pa, Ti, Ww, Yg \\
\hline & & V447A & 2014 Nov 22 & 56983.17 & 8.4 & 254.4 & At, Cd, Ho, Mp \\
\hline \multirow[t]{3}{*}{ GRS 1716-249 } & \multirow[t]{3}{*}{ LBA } & V447D & 2017 Feb 21 & 57805.41 & 8.4 & 283.0 & At, Cd, Ho, Mp \\
\hline & & V447E & 2017 Apr 22 & 57865.73 & 8.4 & 329.3 & At, Cd, Ho, Mp \\
\hline & & V447F & 2017 Aug 13 & 57978.48 & 8.4 & 279.5 & At, Cd, Ho, Ke, Mp, Pa, Td, Ti, Wa, Yg \\
\hline \multirow[t]{10}{*}{ Swift J1753.5-0127 } & \multirow[t]{4}{*}{ VLBA } & BM331A & 2009 Dec 16 & 55181.81 & 8.4 & 149.7 & $\mathrm{Br}, \mathrm{Fd}, \mathrm{Hn}, \mathrm{Kp}, \mathrm{La}, \mathrm{Mk}, \mathrm{Nl}, \mathrm{Ov}, \mathrm{Pt}, \mathrm{Sc}$ \\
\hline & & BM331B & 2009 Dec 19 & 55184.79 & 8.4 & 147.9 & $\mathrm{Br}, \mathrm{Fd}, \mathrm{Hn}, \mathrm{Kp}, \mathrm{La}, \mathrm{Mk}, \mathrm{Nl}, \mathrm{Ov}, \mathrm{Pt}, \mathrm{Sc}$ \\
\hline & & BM331C & 2009 Dec 22 & 55187.81 & 8.4 & 148.6 & $\mathrm{Br}, \mathrm{Fd}, \mathrm{Hn}, \mathrm{Kp}, \mathrm{La}, \mathrm{Mk}, \mathrm{Nl}, \mathrm{Ov}, \mathrm{Pt}, \mathrm{Sc}$ \\
\hline & & BM331D & 2009 Dec 24 & 55189.79 & 8.4 & 147.9 & $\mathrm{Br}, \mathrm{Fd}, \mathrm{Hn}, \mathrm{Kp}, \mathrm{La}, \mathrm{Mk}, \mathrm{Nl}, \mathrm{Ov}, \mathrm{Pt}, \mathrm{Sc}$ \\
\hline & $\mathrm{VLBA}+\mathrm{GBT}$ & BM326 & 2010 Mar 30 & 55285.47 & 8.4 & 189.1 & $\mathrm{Br}, \mathrm{Fd}, \mathrm{Gb}, \mathrm{Hn}, \mathrm{Kp}, \mathrm{La}, \mathrm{Mk}, \mathrm{Nl}, \mathrm{Ov}, \mathrm{Pt}, \mathrm{Sc}$ \\
\hline & \multirow[t]{5}{*}{$\mathrm{EVN}$} & EM101A & 2012 Nov 13 & 56245.03 & 5.0 & 156.9 & Ef, Jb, Mc, Nt, On, Tr, Ys, Wb, Hh \\
\hline & & EM101B & 2013 Mar 20 & 56371.18 & 5.0 & 117.4 & Ef, Jb, Mc, Nt, On, Tr, Ys, Wb, Hh \\
\hline & & EM101C & 2013 Jun 18 & 56461.99 & 5.0 & 174.2 & Ef, Jb, Mc, Nt, On, Tr, Ys, Wb, Hh \\
\hline & & EM101D & 2013 Sep 17 & 56552.74 & 5.0 & 192.1 & $\mathrm{EF}, \mathrm{Jb}, \mathrm{Mc}, \mathrm{Nt}, \mathrm{On}, \mathrm{Tr}, \mathrm{Ys}, \mathrm{Wb}, \mathrm{Hh}$ \\
\hline & & EM101E & 2013 Dec 03 & 56629.75 & 5.0 & 220.8 & Ef, Jb, Mc, Nt, On, Tr, Ys, Wb, Hh \\
\hline
\end{tabular}

Table 2. Information about the calibrators used. The R. A. and Dec. positions of the calibrators are those mentioned in the Astrogeo VLBI calibrator search website http://astrogeo.org/calib/search.html (rfc2019a catalogue). The integrated flux density is the average of the cleaned flux of all epochs of the calibrator. $\theta_{\text {sep }}$ is the angular separation of the target from the calibrator.

\begin{tabular}{lcccccl}
\hline \hline Target & Array & Calibrators & $\begin{array}{c}\text { R. A. (J2000) } \\
(\mathrm{h} \mathrm{m} \mathrm{s})\end{array}$ & $\begin{array}{c}\text { Dec. (J2000) } \\
\left({ }^{\circ} \prime^{\prime \prime}\right)\end{array}$ & $\begin{array}{c}\theta_{\text {sep }} \\
\circ\end{array}$ & $\begin{array}{l}\text { Integrated flux density } \\
(\mathrm{Jy})\end{array}$ \\
\hline GX 339-4 & LBA & $\mathrm{J} 1711-5028$ & $17^{\mathrm{h}} 11^{\mathrm{m}} 40^{\mathrm{s}} .9927$ & $-50^{\circ} 28^{\prime} 17^{\prime \prime} .409$ & 2.21 & 0.08 \\
GRS 1716-249 & LBA & $\mathrm{J} 1711-2509$ & $17^{\mathrm{h}} 11^{\mathrm{m}} 23^{\mathrm{s}} .1020$ & $-25^{\circ} 09^{\prime} 01^{\prime \prime} .564$ & 1.87 & 0.1 \\
Swift J1753.5-0127 & VLBA & $\mathrm{J} 1743-0350$ & $17^{\mathrm{h}} 43^{\mathrm{m}} 58^{\mathrm{s}} .8591$ & $-03^{\circ} 50^{\prime} 04^{\prime \prime} .617$ & 3.36 & 3 \\
& EVN & $\mathrm{J} 1743-0350$ & $17^{\mathrm{h}} 43^{\mathrm{m}} 58^{\mathrm{s}} .8591$ & $-03^{\circ} 50^{\prime} 04^{\prime \prime} .617$ & 3.36 & 2.4 \\
& & $\mathrm{~J} 1752-0147$ & $17^{\mathrm{h}} 52^{\mathrm{m}} 18^{\mathrm{s}} .3640$ & $-01^{\circ} 47^{\prime} 16^{\prime \prime} .685$ & 0.45 & 0.14 \\
\hline
\end{tabular}

finished brief softening periods (Bassi et al. 2019). Ceduna did not have valid data for three hours of observations for the first epoch. The last epoch was obtained during a scheduled VLBI run and hence had access to all telescopes in the LBA other than Hartebeesthoek.

\subsection{Swift J1753.5-0127}

Swift J1753.5-0127 is a high Galactic latitude system which was first discovered when it went into outburst in 2005 (Palmer et al. 2005). It has one of the shortest known orbital periods $(3.2443 \pm 0.001 \mathrm{hr}$ ) for a BHXB (Zurita et al. 2008). This system remained in outburst for 11 years before it ultimately faded to quiescence in 2016 November (Russell et al. 2015). The distance to this source is not well known; the best available estimation being $4-8 \mathrm{kpc}$ (Cadolle Bel et al. 2007 ). The mass of the $\mathrm{BH}$ in this system is $>7.4 M_{\odot}$ (Shaw et al. 2016).

Swift J1753.5-0127 was observed using the EVN, the
VLBA and the HSA (High Sensitivity Array) for a total of nine epochs. The four VLBA observations in December 2009 were conducted with the dual $13 / 4-\mathrm{cm}$ recording mode $(2.3$ and $8.4 \mathrm{GHz}$ ), but we only use the $8.4 \mathrm{GHz}$ data to measure the position due to reduced scattering, reduced ionospheric effects that could give rise to systematic errors in astrometry, and higher sensitivity (Table 1). In addition to the standard calibration steps, a global model of the phase calibrator was made by stacking the calibrated data sets of the phase calibrator from all four epochs to prevent minor differences in the model (due to varying uv-coverage) from introducing astrometric systematics. The calibrated data sets of the target from 2008 were also stacked, as the array does not have the capability to detect a week's motion of the system. Swift J1753.5-0127 was detected in the stacked image with a significance of $\sim 5.2 \sigma$. GBT was a part of the HSA in addition to the standard VLBA dishes for the $8.4 \mathrm{GHz}$ observations in March 2010, where the target was strongly detected $(\sim 124 \sigma)$. 
The EVN observations of Swift J1753.5-0127 were taken as part of a parallax measurement campaign (EM101) in 2012-2013, and consisted of 5 epochs taken over a period of one year. The phase calibrator J1743-0350, which was used in the earlier VLBA observations, was suitable for measuring the proper motion of the target but not the parallax, due to its large angular separation $\left(3.36^{\circ}\right)$ from the target. We therefore conducted an X-ray binary calibrator survey on the VLBA (program code BS206) to find closer compact calibrators. We used the multi-phase centre capability of the DiFX software correlator (Deller et al. 2011) to correlate on the positions of all NVSS sources within $30^{\prime}$ of the target, observing at $1.6 \mathrm{GHz}$ to give a field of view such that only four pointings were required to cover the desired sky area. Within $30^{\prime}$ of Swift J1753.5-0127 we detected two potential calibrators, J1752-0147 $\left(17^{\mathrm{h}} 52^{\mathrm{m}} 18.364^{\mathrm{s}},-01^{\circ} 47^{\prime} 16.685^{\prime \prime}\right)$ and J1753-0102 $\left(17^{\mathrm{h}} 53^{\mathrm{m}} 10.4488^{\mathrm{s}},-01^{\circ} 02^{\prime} 48.854^{\prime \prime}\right)$. While the latter turned out to be a widely-separated double, the former was a compact, single source of peak flux density 6-9 mJy, and was therefore adopted as a secondary phase calibrator for our EVN parallax campaign, and was $0.44^{\circ}$ away from our target.

To make sure that we were using the same source models to solve for the delays and rates, we concatenated the calibrated data sets of the primary calibrator for all epochs. This model was then used to derive (epoch-wise) the phase, delay and rate solutions that were interpolated to the secondary calibrator and the target. The secondary calibrator was then imaged and the data were stacked to obtain a global model (as done by Miller-Jones et al. 2018). The global model was again used to derive epoch-wise phase solutions that were applied to the target data, which were then imaged. The target positions were then measured by fitting a point source model in the image plane.

\section{RESULTS - PROPER MOTION MEASUREMENTS}

Our VLBI data were used to find the proper motions of GX 339-4 and GRS 1716-249 for the first time. We also measured the proper motion of Swift J1753.5-0127, for which Gaia also had made a measurement. The position measurements of the three sources at all epochs are summarised in Table 3 .

\subsection{GX 339-4}

As shown in Figure 1, for GX 339-4 a linear fit for the proper motion of the system gives:

$$
\begin{aligned}
\mu_{\alpha} \cos \delta & =-3.95 \pm 0.07 \text { mas yr}^{-1} \\
\mu_{\delta} & =-4.71 \pm 0.06 \text { mas yr}^{-1} .
\end{aligned}
$$

This gives an overall proper motion for GX339-4 of

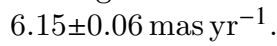

\subsection{GRS $1716-249$}

The measured offsets of GRS 1716-249 in RA and Dec for our three epochs of LBA observations have been plotted in

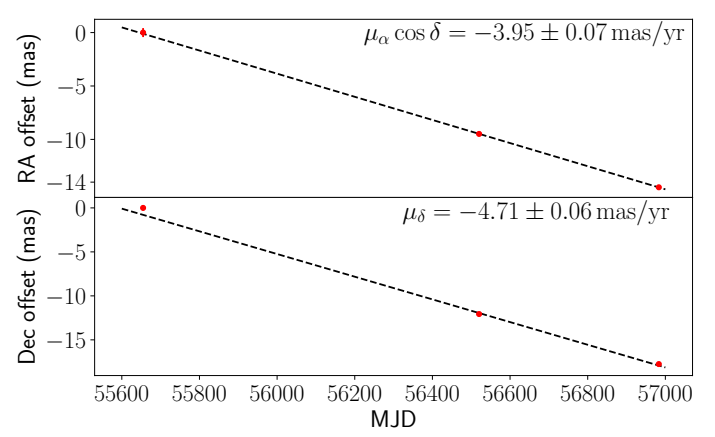

Figure 1. Proper motion fit for GX 339-4. GX 339-4 was tracked for three epochs over three different outbursts. Top panel: Offset of measured positions relative to the first epoch in Right Ascension. Bottom panel: Offset of measured positions in Declination relative to the first epoch.
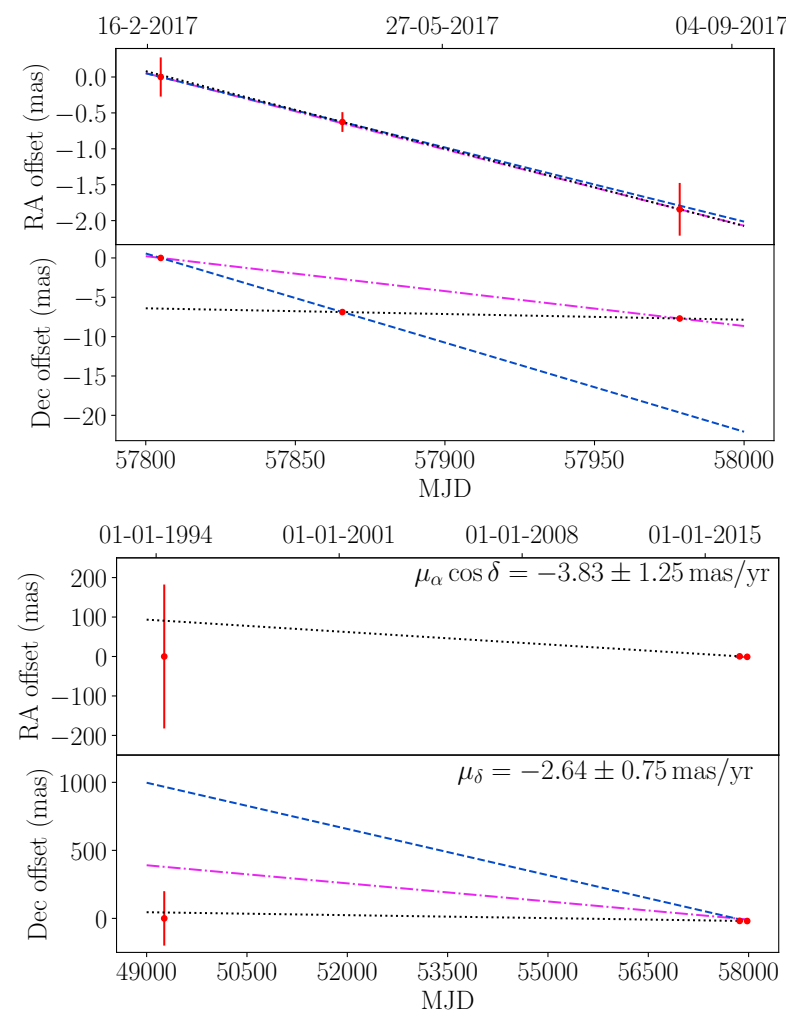

Figure 2. Proper motion fits for GRS 1716-249. GRS 1716-249 was tracked for three epochs over one outburst using the LBA, with positions shown with respect to the first LBA epoch. Top panel: fits by considering position measurements of two epochs at a time in RA and Dec - epoch 1 and 2 (dashed line); epoch 2 and 3 (dotted line); epoch 1 and 3 (dotted dashed line). The three declination measurements do not lie on a straight line. Lower panel: fitting for proper motion by increasing the time baseline using the $1994 \mathrm{~K}$-band image position measurement (dotted line), which breaks the degeneracy and shows that the epoch 1 LBA position is in error (blue and green dashed lines). This was then removed from the fit. 
Table 3. Summary of the detections of GX 339-4, GRS 1716-249 and Swift J1753.5-0127. MJD is taken as the middle of the observing run and the error on MJD is calculated as half the length of the observation. The positions, peak intensities and errors on each parameter are calculated by fitting an elliptical Gaussian to the target in the image plane.

\begin{tabular}{lccccc}
\hline \hline Target & Project Code & $\begin{array}{c}\text { MJD } \\
\text { (UTC) }\end{array}$ & $\begin{array}{c}\text { R. A. (J2000) } \\
(\mathrm{h} \mathrm{m} \mathrm{s})\end{array}$ & $\begin{array}{c}\text { Dec. (J2000) } \\
\left({ }^{\circ} \prime^{\prime \prime}\right)\end{array}$ & $\begin{array}{c}\text { Peak Intensity } \\
\left.(\mu \mathrm{Jy} \mathrm{bm})^{-1}\right)\end{array}$ \\
\hline GX 339-4 & V430A & $55654.84 \pm 0.25$ & $17^{\mathrm{h}} 02^{\mathrm{m}} 49^{\mathrm{s}} .38260 \pm 0.00004$ & $-48^{\circ} 47^{\prime} 23^{\prime \prime} .1413 \pm 0.0003$ & $197 \pm 18$ \\
& V486B & $56520.21 \pm 0.24$ & $17^{\mathrm{h}} 02^{\mathrm{m}} 49^{\mathrm{s}} .38164 \pm 0.00001$ & $-48^{\circ} 47^{\prime} 23^{\prime \prime} .1534 \pm 0.0001$ & $2280 \pm 163$ \\
& V447A & $56983.17 \pm 0.16$ & $17^{\mathrm{h}} 02^{\mathrm{m}} 49^{\mathrm{s}} .38114 \pm 0.00001$ & $-48^{\circ} 47^{\prime} 23^{\prime \prime} .1591 \pm 0.0001$ & $2629 \pm 203$ \\
GRS 1716-249 & V447D & $57805.41 \pm 0.31$ & $17^{\mathrm{h}} 19^{\mathrm{m}} 36^{\mathrm{s}} .92008 \pm 0.00002$ & $-25^{\circ} 01^{\prime} 04^{\prime \prime} .1215 \pm 0.0005$ & $1488 \pm 156$ \\
& V447E & $57865.73 \pm 0.23$ & $17^{\mathrm{h}} 19^{\mathrm{m}} 36^{\mathrm{s}} .92003 \pm 0.00001$ & $-25^{\circ} 01^{\prime} 04^{\prime \prime} .1286 \pm 0.0001$ & $1135 \pm 105$ \\
& V447F & $57978.48 \pm 0.19$ & $17^{\mathrm{h}} 19^{\mathrm{m}} 36^{\mathrm{s}} .91994 \pm 0.00003$ & $-25^{\circ} 01^{\prime} 04^{\prime \prime} .1294 \pm 0.0002$ & $290 \pm 42$ \\
& BM331 & $55186.05 \pm 0.10$ & $17^{\mathrm{h}} 53^{\mathrm{m}} 28^{\mathrm{s}} .29060 \pm 0.00001$ & $-01^{\circ} 27^{\prime} 06^{\prime \prime} .2916 \pm 0.0002$ & $290 \pm 56$ \\
& BM326 & $55285.47 \pm 0.14$ & $17^{\mathrm{h}} 53^{\mathrm{m}} 28^{\mathrm{s}} .29061 \pm 0.000003$ & $-01^{\circ} 27^{\prime} 06^{\prime \prime} .2919 \pm 0.0001$ & $2614 \pm 21$ \\
& EM101A & $56245.03 \pm 0.48$ & $17^{\mathrm{h}} 53^{\mathrm{m}} 28^{\mathrm{s}} .29074 \pm 0.00002$ & $-01^{\circ} 27^{\prime} 06^{\prime \prime} .3011 \pm 0.0003$ & $280 \pm 27$ \\
& EM101B & $56371.18 \pm 0.08$ & $17^{\mathrm{h}} 53^{\mathrm{m}} 28^{\mathrm{s}} .29077 \pm 0.00005$ & $-01^{\circ} 27^{\prime} 06^{\prime \prime} .3037 \pm 0.0006$ & $161 \pm 31$ \\
& EM101C & $56461.99 \pm 0.09$ & $17^{\mathrm{h}} 53^{\mathrm{m}} 28^{\mathrm{s}} .29078 \pm 0.00003$ & $-01^{\circ} 27^{\prime} 06^{\prime \prime} .3036 \pm 0.0004$ & $141 \pm 20$ \\
& EM101D & $56552.74 \pm 0.13$ & $17^{\mathrm{h}} 53^{\mathrm{m}} 28^{\mathrm{s}} .29081 \pm 0.00002$ & $-01^{\circ} 27^{\prime} 06^{\prime \prime} .3053 \pm 0.0004$ & $207 \pm 27$ \\
& EM101E & $56629.75 \pm 0.10$ & $17^{\mathrm{h}} 53^{\mathrm{m}} 28^{\mathrm{s}} .29082 \pm 0.00004$ & $-01^{\circ} 27^{\prime} 06^{\prime \prime} .3054 \pm 0.0006$ & $109 \pm 21$ \\
\hline
\end{tabular}

Figure 2. Measured declinations in these epochs do not appear to follow a linear trend (Top panel of Fig. 2). Choosing different subsets of measurements yield drastically different results. The proper motions of the different fits (considering two epochs at a time) in RA agree with each other within the $1 \sigma$ error limit. On the contrary, the proper motion fits

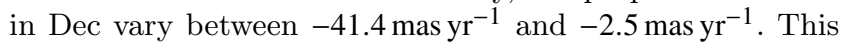
suggests that the measured position in Declination for one out of the three epochs is unreliable.

To ascertain which of the epochs were giving accurate positions, we obtained an archival position measurement of GRS 1716-249 from a near infrared observation to increase the time baseline. We use the position of GRS 1716-249 as measured from the K-band image taken on MJD 49263 during the 1994 outburst (Chaty et al. 2002). We fixed the image astrometry to the Gaia-DR2 frame by cross matching it with $\sim 140$ sources from the Gaia-DR2 catalogue using PyRAF. The proper motions of these sources as reported by Gaia-DR2 were used to correct the positions of the sources. The position of GRS 1716-249 was determined with an uncertainty of $\sim 0.2$ arcsec. This shows that the position from the first LBA epoch is the cause of the discrepancy. As mentioned in Section 2.2, Ceduna was not available for observing during most of that observing run. Thus the uv coverage was sparse and could explain the wrong position measurement. To determine the true proper motion, we therefore fit using the last two LBA epochs (V447E and V447F) and the position measurement from the 1994 K-band image. We obtain the following proper motion

$$
\begin{aligned}
\mu_{\alpha} \cos \delta & =-3.83 \pm 1.25 \text { mas yr}^{-1} \\
\mu_{\delta} & =-2.64 \pm 0.75 \text { mas yr }^{-1} .
\end{aligned}
$$

This gives an overall proper motion of $4.65 \pm 1.12{\text { mas } \mathrm{yr}^{-1}}^{-1}$ for GRS 1716-249.

\subsection{Swift J1753.5-0127}

Figure 3 shows a linear fit to the measured positions of Swift J1753.5-0127 over a span of 4 years. Although we used a different calibration scheme for the VLBA and EVN

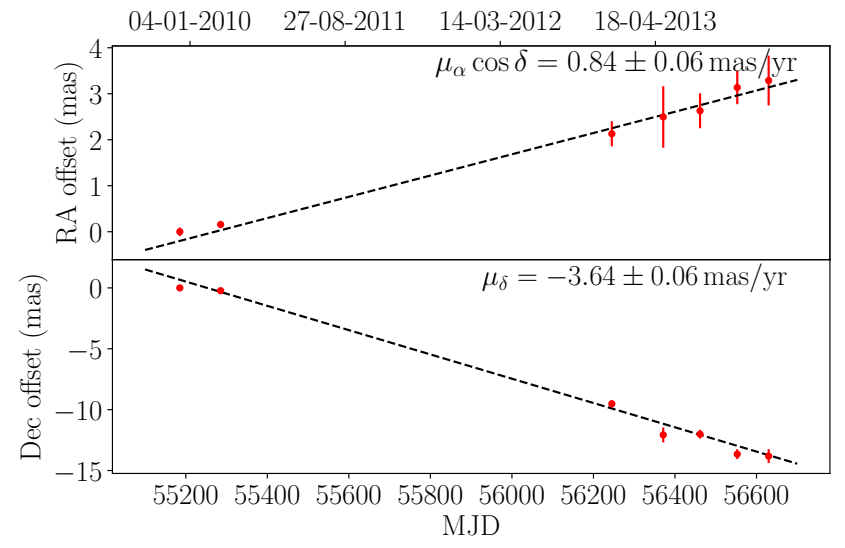

Figure 3. Proper motion fit for Swift J1753.5-0127. Swift J1753.5-0127 was observed for ten epochs spanning four years. The four observations in 2009 December were concatenated into one position measurement (MJD 55181 to MJD 55190). Top panel: offset of measured positions in Right Ascension of Swift J1753.5-0127 as a function of MJD relative to the first epoch. Bottom panel: offset of measured positions in Declination of Swift J1753.5-0127 as a function of MJD relative to the first epoch.

epochs, the nearby secondary calibrator used for the EVN (J1752-0147) was observed using the VLBA primary calibrator J1743-0350 as a phase reference source, such that the two data sets should be referenced to the same absolute reference frame. The absolute astrometric systematics should therefore be of order 0.09 and 0.27 mas, respectively in R.A. and Dec. The fit gives a proper motion of

$$
\begin{gathered}
\mu_{\alpha} \cos \delta=0.84 \pm 0.06 \mathrm{mas} \mathrm{yr}^{-1} \\
\mu_{\delta}=-3.64 \pm 0.06 \mathrm{mas} \mathrm{yr}^{-1} .
\end{gathered}
$$

This gives a net proper motion of $3.73 \pm 0.06 \mathrm{mas}^{-1}$. The proper motion determined above agrees within $2 \sigma$ error lim- 
its with that reported by Gaia-DR2, which is

$$
\begin{gathered}
\mu_{\alpha} \cos \delta=1.13 \pm 0.16 \text { mas yr}^{-1} \\
\mu_{\delta}=-3.53 \pm 0.15 \text { mas yr}^{-1} .
\end{gathered}
$$

\section{DISTANCE AND RADIAL VELOCITY ESTIMATES}

We will fold the proper motions measured by our VLBI data for the three systems as reported in Section 4, and those available in archival data (using VLBI and Gaia) with distance and systemic radial velocities of these systems to get their potential kick velocity distribution.

\subsection{Distance - Milky way prior}

The distances to some of the sources in our sample have been inferred from a parallax measurement made by GaiaDR2 (Gaia Collaboration et al. 2018) or VLBI. Every Gaia measurement of a parallax has an uncertainty associated with it. The fractional errors associated with these measurements are significant, even if they are small in absolute value. Thus, it is essential that these errors are properly considered when converting parallax to distance. In order to make sure that the errors from the measured quantity are appropriately propagated to the inferred quantity, we use a Bayesian inference approach (Astraatmadja \& Bailer-Jones 2016).

We use the likelihood function as described in BailerJones (2015) and Gandhi et al. (2019). We follow the work by Grimm et al. (2002, GR02 hereafter) to make an analytical model of the LMXB density distribution in our Galaxy to use as the prior. This considers the density of the bulge, disc and spheroid of the Galaxy, as described by the following equations (equations 4,5,6 in GR02).

$$
\begin{aligned}
& \rho_{\text {Bulge }}=\rho_{0, \text { Bulge }} \cdot\left(\frac{\sqrt{r^{2}+\frac{z^{2}}{q^{2}}}}{r_{0}}\right)^{-\gamma} \cdot \exp \left(-\frac{r^{2}+\frac{z^{2}}{q^{2}}}{r_{\mathrm{t}}^{2}}\right), \\
& \rho_{\text {Disk }}=\rho_{0, \text { Disk }} \cdot \exp \left(-\frac{\mathrm{r}_{\mathrm{m}}}{\mathrm{r}_{\mathrm{d}}}-\frac{\mathrm{r}}{\mathrm{r}_{\mathrm{d}}}-\frac{|\mathrm{z}|}{\mathrm{r}_{\mathrm{z}}}\right), \\
& \rho_{\text {Sphere }}=\rho_{0, \text { Sphere }} \cdot \frac{\exp \left(-\mathrm{b} \cdot\left(\frac{\mathrm{R}}{\mathrm{R}_{\mathrm{e}}}\right)^{\frac{1}{4}}\right)}{\left(\frac{\mathrm{R}}{\mathrm{R}_{\mathrm{e}}}\right)^{\frac{7}{8}}} .
\end{aligned}
$$

Here $r$ is the distance from the Galactic centre of the projected position of the source on the Galactic plane, $z$ is the height of the source above the Galactic plane, and $R$ is the distance of the source from the Galactic centre. $q, r_{0}, r_{\mathrm{t}}, r_{\mathrm{d}}$, $r_{\mathrm{m}}, r_{\mathrm{z}}$ and $R_{\mathrm{e}}$ are scale parameters. GR02 derived these scale parameters for LMXBs by constructing the X-ray luminosity function of these systems. We use the values of these constants as summarised in Table 4 of GR02. $\rho_{0 \text {, Bulge }}, \rho_{0 \text {,Disk }}$ and $\rho_{0, \text { Sphere }}$ are normalisation constants. We estimate the density constants $\rho_{0 \text {,Bulge }}, \rho_{0 \text {,Disk }}$ and $\rho_{0 \text {,Sphere }}$ for LMXBs using the Disk:Bulge:Sphere mass ratio of $2: 1: 0.5$ as derived by GR02, and assuming the mass of the bulge as $1.3 \times 10^{10} \mathrm{M}_{\odot}$ (GR02). This gave the values of $\rho_{0 \text {, Bulge }}, \rho_{0 \text {,Disk }}$ and $\rho_{0 \text {, Sphere }}$ as $1.1 \mathrm{M}_{\odot} \mathrm{pc}^{-3}, 2.6 \mathrm{M}_{\odot} \mathrm{pc}^{-3}$ and $13.1 \mathrm{M}_{\odot} \mathrm{pc}^{-3}$ respectively. We note that the spatial distribution model of GR02 is not exclusively for BHXBs as their work considered neutron star
X-ray binaries as well, but GR02 is currently the best available model for the spatial distribution of X-ray binaries as existing stellar spatial distributions do not account for kicks.

The parallaxes measured by Gaia-DR2 have a global zero-point offset of -0.029 mas (Luri et al. 2018). Hence the parallax values from Gaia were corrected before being used for the distance estimation using the Milky Way prior. These corrected parallax values along with the estimated values of distances of 11 BHXBs for which Gaia-DR2 measured parallaxes has been reported in Table 4 and Table 5. There are also reports that the Gaia-DR2 parallaxes on average have a systematic offset of $-0.075 \pm 0.029$ mas (Xu et al. 2019), but we use the more established -0.029 mas offset (Luri et al. 2018) for the correction of the parallaxes. 11 of the 16 systems in our sample also have distance estimations in the literature (see Table 4 and 5 for more details), and Gaia-DR2 measured the parallax of six of those systems (1A 0620-00, XTE J1118+480, GS 1124-684, GRO J1655-40, Swift J1753.5-0127 and SAX J1819-2525). We compare the distance estimates derived from Gaia parallaxes to the ones available in the literature and use the better constrained distance for determining and analysing the PKV distributions.

\subsection{Systemic radial velocity estimates}

We have four systems with poorly constrained systemic radial velocities (Swift J1753.5-0127, MAXI J1820+070, VLA J2130+12 and GRS 1716-249). Using the best distance estimates for these systems, we project them onto the Galactic plane. We estimate the expected systemic radial velocity $(\bar{\gamma})$ of the system at this projected distance onto the Galactic plane and assuming that the system is undergoing pure Galactic rotation about the Galactic centre. We note that this estimated value of systemic radial velocity is not a true indicator of the systemic radial velocity of the system as it might have received kicks, and thus is probably not following a pure Galactic rotation about the Galactic centre. Thus for each system, we estimate five probable systemic radial velocity Gaussian distributions with medians of $\bar{\gamma}, \bar{\gamma} \pm$ $50 \mathrm{~km} \mathrm{~s}^{-1}$ and $\bar{\gamma} \pm 100 \mathrm{~km} \mathrm{~s}^{-1}$ (see Table 5). We limit our assumed distributions of the systemic radial velocities based on the fact that out of the systems for which systemic radial velocity is measured, the values lie between $-142 \pm 1.5 \mathrm{~km} \mathrm{~s}^{-1}$ for GRO J1655-40 (Shahbaz et al. 1999) and 107 $\pm 2.9 \mathrm{~km} \mathrm{~s}^{-1}$ for SAX J1819.3-2525 (Orosz et al. 2001).

\section{ANALYSIS - POTENTIAL KICK VELOCITY}

As explained in Section 1.3, peculiar velocity of a system when it crosses the Galactic plane is a better probe to understand the kick a BHXB received when the BH was born. Since all the parameters, namely proper motion, systemic radial velocity and parallax (or distance), have error bars associated with them, it is crucial to propagate these errors appropriately to estimate the Galactocentric orbits of the systems. The age of most BHXBs is not known, which makes integrating the Galactocentric orbits back to the time of birth of the $\mathrm{BH}$ uncertain. We thus developed a Monte Carlo (MC) methodology that accounts for the errors on the 
measured quantities and determines the peculiar velocity every time the system crosses the Galactic plane.

The code integrates the Galactocentric orbit of every system in our sample back for 10 Gyrs and records the velocity of the system at every plane crossing. Instead of a delta function for the measurement, it involves using Gaussian distributions of the measured parameters with reported uncertainties as standard deviation. Random values are picked from these Gaussian distributions as inputs to galpy (Bovy 2014 ) to create instances of Galactocentric orbits for $\sim 5000$ random draws to make sure we have sampled the input distribution properly. We assume a Galactic potential given by the galpy model MWPotential2014 (see Bovy 2014). The Galactocentric orbits are integrated back in time for 10 Gyrs, which exceeds the likely ages of LMXB systems. GRO J1655-40 (Shahbaz 2003), GS 1354-64 (Casares et al. 2009) and SAX J1819-2525 (MacDonald et al. 2014) are intermediate-mass systems and may have a shorter lifetime than standard LMXBs. Cyg X-1 is a HMXB and has not lived long enough to cross the Galactic plane (Wong et al. 2012). So this calculation, while indicative of the kick, does not represent the system's true history. To check if the integration of the systems back for 10 Gyrs is valid for these systems, we integrated the orbits of these systems back in time for $1 \mathrm{Gyr}$ with 50,000 random draws and found that our results were not affected and were the same as those using 5000 draws and 10 Gyr orbits. Thus, for uniformity we have used a time integration of 10 Gyrs for all systems.

The peculiar velocity at each Galactic plane crossing (i.e. $\mathrm{z}=0$, where $\mathrm{z}$ is the height above the Galactic plane) is calculated as

$v_{\text {peculiar }}=\left[\left(U-U_{0}\right)^{2}+\left(V-V_{0}\right)^{2}+\left(W-W_{0}\right)^{2}\right]^{0.5}$

Here $U, V$ and $W$ are Galactic space velocities towards the Galactic centre, in the direction of Galactic rotation and towards the North Galactic Pole respectively (Johnson \& Soderblom 1987). $U_{0}, V_{0}$ and $W_{0}$ are the $U, V, W$ components of the Galactocentric space velocities of the local standard of rest at a time when the system crosses the Galactic plane.

This approach allows us to estimate potential kick velocity distributions even if all the parameters required to construct the three dimensional motion are not accurately known. This will also help in estimating the potential kick velocities for newly discovered BHXBs that go into outburst, and when uncertain parameters of known systems are updated with new measurements. The code detailing the MC simulation methodology for estimating the potential kick velocity probability distribution and the distance estimation using our Milky way prior is available at the github link https://github.com/pikkyatri/BHnatalkicks.

\subsection{Potential kick velocity distributions of the new VLBI sources}

We estimated the potential kick velocity (PKV) probability distributions for the three sources for which we made proper motion measurements; GX 339-4, Swift J1753.5-0127 and GRS 1716-249. The proper motion input for the MC simulation is a Gaussian distribution based on the measured proper motion and error bars.

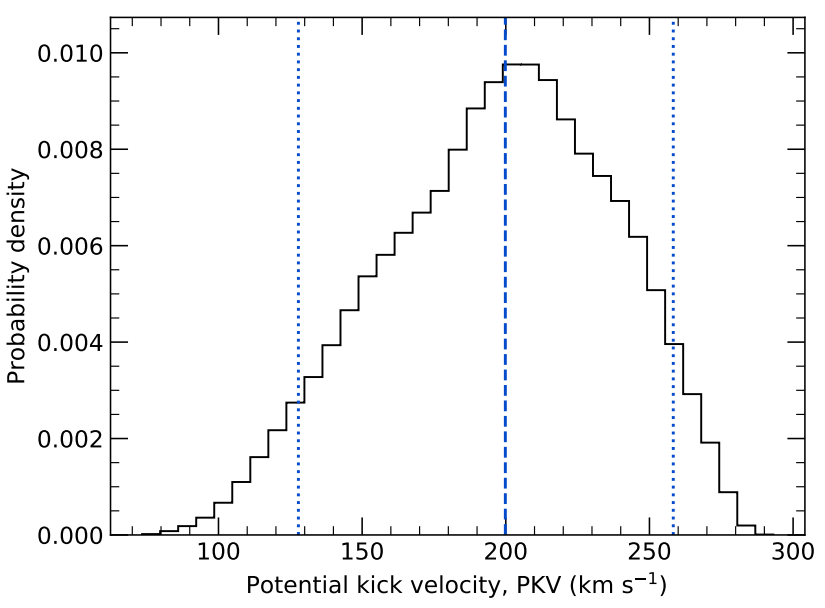

Figure 4. Potential kick velocity probability distribution of GX 339-4 with a median of $200 \mathrm{~km} \mathrm{~s}^{-1}$ (dashed line) and a $5^{\text {th }}$ and $95^{\text {th }}$ percentile of $122 \mathrm{~km} \mathrm{~s}^{-1}$ and $258 \mathrm{~km} \mathrm{~s}^{-1}$ (in dotted lines), respectively.

\subsubsection{GX 339-4}

Gaia could not make a parallax measurement for GX 339-4. For the distance input we used a uniform distribution between 5 and $13 \mathrm{kpc}$ as estimated by Heida et al. (2017). The system velocity input for the code was a Gaussian distribution based on the measurement of Heida et al. (2017) (Table 4). Running MC simulations for these distributions gives a PKV probability distribution as shown in Figure 4 with a median of $200 \mathrm{~km} \mathrm{~s}^{-1}$ snd the $5^{\text {th }}$ percentile at $122 \mathrm{~km} \mathrm{~s}^{-1}$.

\subsubsection{GRS 1716-249}

della Valle et al. (1994) estimated the distance to GRS 1716249 as $2.4 \pm 0.4 \mathrm{kpc}$, which we used as a Gaussian input prior. The systemic radial velocity of this system has not been measured yet. A system at the Galactocentric radius of GRS 1716-249 and in the Galactic plane is expected to have a systemic radial velocity $\bar{\gamma}$ of $\sim-10 \mathrm{~km} \mathrm{~s}^{-1}$. We thus run the MC simulations assuming five different Gaussian systemic radial velocity distributions with means of $110 \mathrm{~km} \mathrm{~s}^{-1},-60 \mathrm{~km} \mathrm{~s}^{-1},-10 \mathrm{~km} \mathrm{~s}^{-1}, 40 \mathrm{~km} \mathrm{~s}^{-1}$ and $90 \mathrm{~km} \mathrm{~s}^{-1}$, all with a $1 \sigma$ of $50 \mathrm{~km} \mathrm{~s}^{-1}$. All five PKV probability distributions have a median above $\sim 70 \mathrm{~km} \mathrm{~s}^{-1}$ (see Figure 5, left panel and Table 4). In figure 5 (right panel) we plotted twenty orbits for the maximum $\left(100_{-47}^{+68} \mathrm{~km} \mathrm{~s}^{-1}\right)$ and minimum $\left(67_{-27}^{+41} \mathrm{~km} \mathrm{~s}^{-1}\right)$ PKVs corresponding to systemic radial velocities of $90 \mathrm{~km} \mathrm{~s}^{-1}$ and $-10 \mathrm{~km} \mathrm{~s}^{-1}$, respectively. The orbits stay within the vertical thick stellar disc limits of $\sim 1 \mathrm{kpc}$ (Gilmore \& Reid 1983).

\subsubsection{Swift J1753.5-0127}

Gaia-DR2 measured a parallax of $-0.01 \pm 0.13$ mas for this system. As there is a zero-point offset in all Gaia parallax measurements of -0.029 mas (Luri et al. 2018), we use the corrected parallax of $0.02 \pm 0.13$ mas for our simulations. We determined a posterior distribution for the distance as shown 

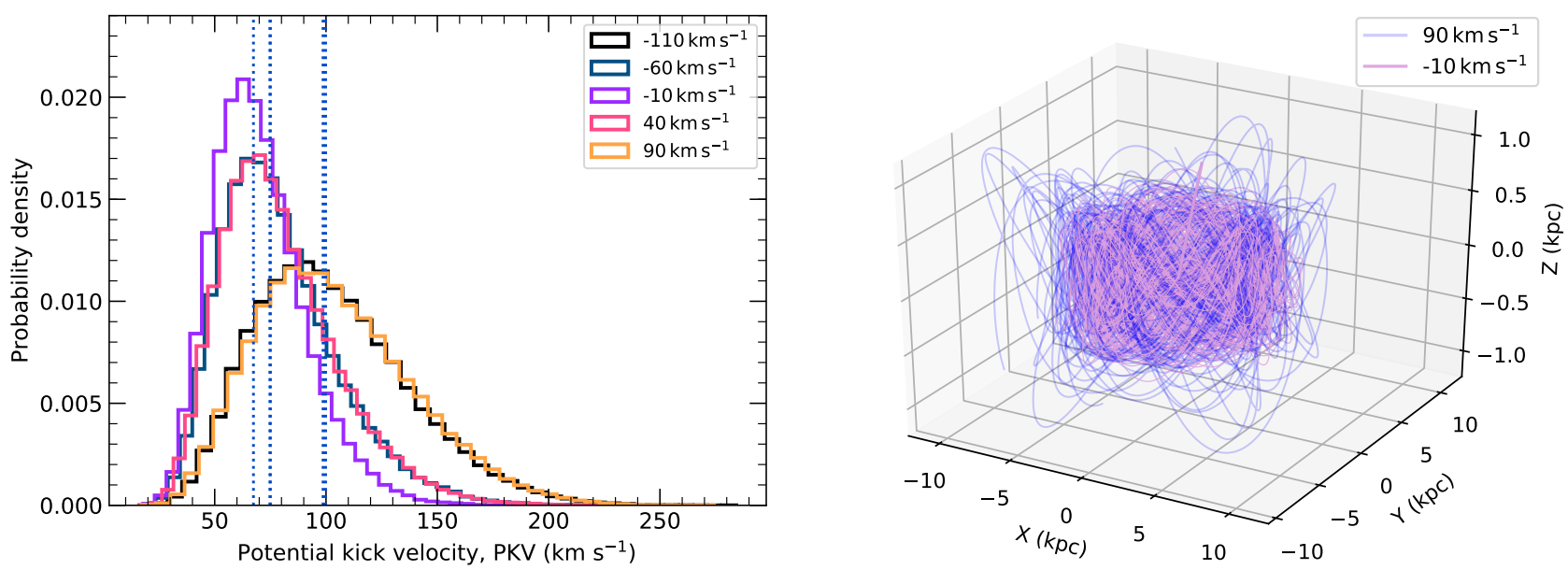

Figure 5. Left panel: PKV probability distribution of GRS 1716-249 using Gaussian systemic radial velocity $(\bar{\gamma})$ distributions with means of $-110 \mathrm{~km} \mathrm{~s}^{-1},-60 \mathrm{~km} \mathrm{~s}^{-1},-10 \mathrm{~km} \mathrm{~s}^{-1}, 40 \mathrm{~km} \mathrm{~s}^{-1}$ and $90 \mathrm{~km} \mathrm{~s}^{-1}$ all with a $1 \sigma$ of $50 \mathrm{~km} \mathrm{~s}^{-1}$. The medians of all the PKV distributions are the blue dashed vertical lines. The lowest PKV probability distribution median is of $\sim 70 \mathrm{~km} \mathrm{~s}^{-1}$ and corresponds to the systemic radial velocity of $-10 \pm 50 \mathrm{~km} \mathrm{~s}^{-1}$. Right panel: a 3-D visualisation of the Galactocentric orbit of GRS 1716-249, integrated for 1 Gyr for 20 orbit instances each of the lowest $\left(67_{-27}^{+41} \mathrm{~km} \mathrm{~s}^{-1}\right)$ and highest $\left(100_{-47}^{+68} \mathrm{~km} \mathrm{~s}^{-1}\right)$ PKV corresponding to systemic radial velocities of $-10 \pm 50 \mathrm{~km} \mathrm{~s}{ }^{-1}$ and $90 \pm 50 \mathrm{~km} \mathrm{~s}^{-1}$, respectively. All three axes are in kpc. The system does not go beyond $1 \mathrm{kpc}$ above the Galactic plane in both the cases.
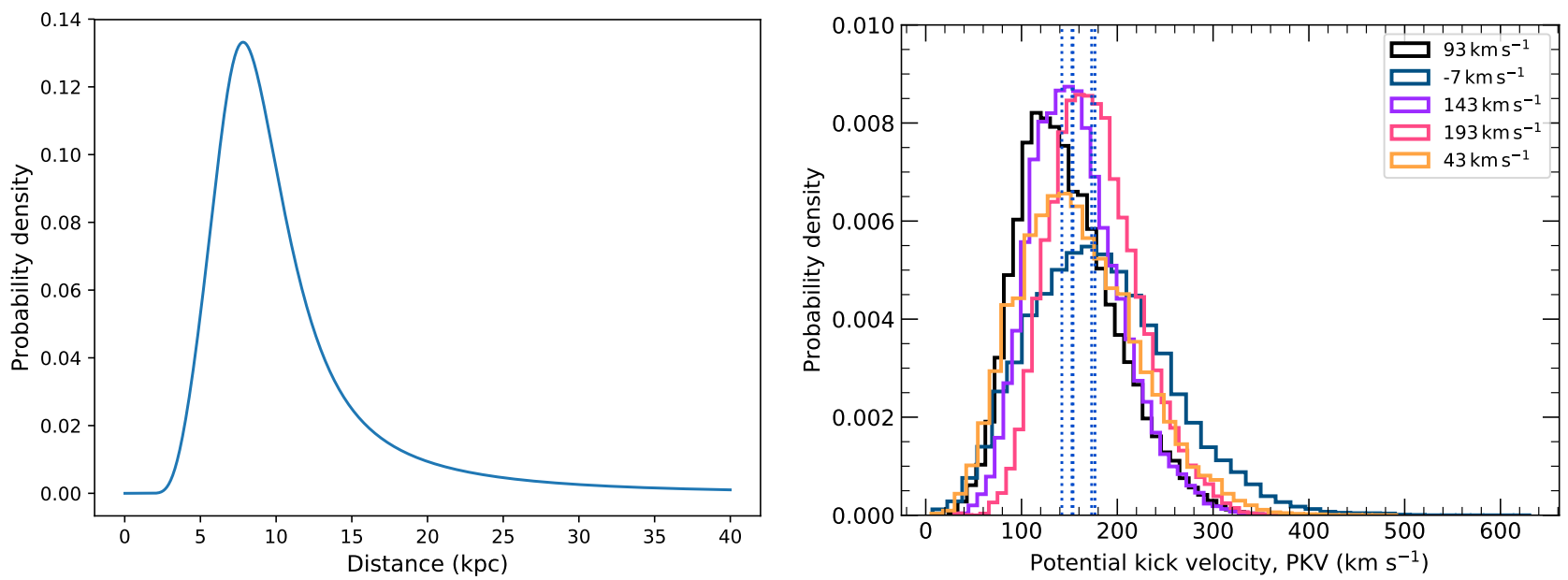

Figure 6. Left panel: Posterior distribution function for the distance to Swift J1753.5-0127, constructed from the Gaia-DR2 parallax measurement using the Milky Way LMXB distribution prior (see Section 5.1 for details). The mode of the distribution is at $7.7 \mathrm{kpc}$. The $5^{\text {th }}, 50^{\text {th }}$ and $95^{\text {th }}$ percentiles of the distance are at $4.8,8.8$ and $20.8 \mathrm{kpc}$ respectively. Right panel: PKV probability distribution of Swift J1753.5-0127 using Gaussian systemic radial velocity $(\bar{\gamma})$ distributions with means of $-7 \mathrm{~km} \mathrm{~s}^{-1}, 43 \mathrm{~km} \mathrm{~s}^{-1}, 93 \mathrm{~km} \mathrm{~s}^{-1}, 143 \mathrm{~km} \mathrm{~s}^{-1}$ and $193 \mathrm{~km} \mathrm{~s}^{-1}$ all with a $1 \sigma$ of $50 \mathrm{~km} \mathrm{~s}^{-1}$. The input distance distribution of $6 \pm 2 \mathrm{kpc}$ was used instead of the distance posterior distribution derived using the Gaia parallax as the former was more tightly constrained. The lowest median amongst all the PKV probability distributions is at $142 \mathrm{~km} \mathrm{~s}^{-1}$ and the $5^{\text {th }}$ percentiles of all the PKV probability distributions are above $70 \mathrm{~km} \mathrm{~s}^{-1}$.

in Figure 6-Left panel. Comparing this with the distance estimated for this source in the literature $(6 \pm 2 \mathrm{kpc}$ ) (Cadolle Bel et al. 2007) suggests that the poorly constrained Gaia parallax may be overestimating the distance. We thus use a Gaussian distance distribution centred at $6 \mathrm{kpc}$ with a $1 \sigma$ of $2 \mathrm{kpc}$ as input for the simulations rather than the distance distribution derived using Gaia parallax. Along with the distance distribution, we use Gaussian distributions of the proper motion and five different Gaussian distributions (see Section 5.2 and Table 5) as systemic radial velocity $(\gamma)$ inputs to calculate the PKV probability distribution (Figure 6-Right panel). Even the lowest median amongst the five PKV probability distributions is at $142 \mathrm{~km} \mathrm{~s}^{-1}$, with the $5^{\text {th }}$ and $95^{\text {th }}$ percentiles as $76 \mathrm{~km} \mathrm{~s}^{-1}$ and $243 \mathrm{~km} \mathrm{~s}^{-1}$, respectively (see Table 4 ). Over the past 10 Gyrs, the system has reached as high as $1.5 \mathrm{kpc}$ above the Galactic plane. 


\subsection{PKV distributions of Gaia DR-2 and archival sources}

We used our methodology (see Section 5.1) to invert the Gaia-DR2 parallaxes for nine systems that Gaia-DR2 measured parallaxes, and for two sources (V404 Cyg and VLA J2130+12) that had archival parallax measurements . We have summarised the results for these simulations in Table 4 . Out of these 11 systems, seven also had distance constraints in the literature. We used the distance estimations from the literature $\left(d_{\text {lit }}\right)$ as inputs for the MC simulations to obtain another set of PKV probability distributions for these seven systems (1A 0620-00, GS 1124-684, GRO J165540， Swift J1753.5-0127， SAX J1819-2525， XTE J1118+480 and MAXIJ1820+070). We thus obtain two PKV probability distributions each for these seven systems, but use the PKV probability distributions determined using the literature distance estimates $\left(d_{\text {lit }}\right)$ for further analysis of these systems as they were more tightly constrained.

We used the distance posterior derived from parallax measurements for our PKV probability distributions for four systems, namely GS 1354-64, Cyg X-1, V404 Cyg and VLA J2130+12, as they did not have better distance estimates in the literature (See Table 4 and Table 5). We tested the sensitivity of the PKV distribution of these four systems to the prior we are using to calculate the distance posterior, using an exponentially decreasing volume density prior (Astraatmadja \& Bailer-Jones 2016; Gandhi et al. 2019) to determine a revised PKV distribution. The only system showing a marked difference in the PKV distributions from the two priors was GS 1354-64, with a revised median PKV of $183_{-64}^{+102} \mathrm{~km} \mathrm{~s}^{-1}$ as compared to $213_{-78}^{+88} \mathrm{~km} \mathrm{~s}^{-1}$ from the Milky Way prior. However, even this difference was within the uncertainties of the PKV distribution using the Milky Way prior.

For V404 Cyg, we use the archival VLBI proper motion and parallax measurements (Miller-Jones et al. 2009a) as they were more precise than the Gaia-DR2 measurements. The parallax and proper motion for VLA J2130+12 were measured by Kirsten et al. (2014), though there is no available estimate of its systemic radial velocity. We thus used the expected radial velocity of a source at the Galactocentric distance of VLA J2130+12 but in the Galactic plane as the input for estimating its PKV probability distribution. For systems that did not have measured parallaxes, we use the best estimates on distance present in the literature (Table 5). Gaia-DR2 did not measure proper motions for MAXI J1836-194 and GRS 1915+105, so we also use archival VLBI proper motion measurements for these systems. We have reported the $5^{t h}, 50^{\text {th }}$ and $95^{\text {th }}$ percentiles for both the distance distribution that we determined using the LMXB Milky Way prior described in Section 5.1, and for the potential kick velocities for these systems.

MAXI J1820+070 was first detected in March 2018 (Kawamuro et al. 2018) and is still being monitored in the radio and X-ray bands. We obtained an upper limit on the distance of the system as $3.9 \pm 0.6 \mathrm{kpc}$ by constraining the proper motions of the receding and approaching jets observed in existing VLA data (Bright et al. in prep). For a lower limit on the distance, we used the X-ray flux reported by Swift at the soft-to-hard X-ray spectral state transition. We assume that the state transition happens between $1 \%$ and $4 \%$ of

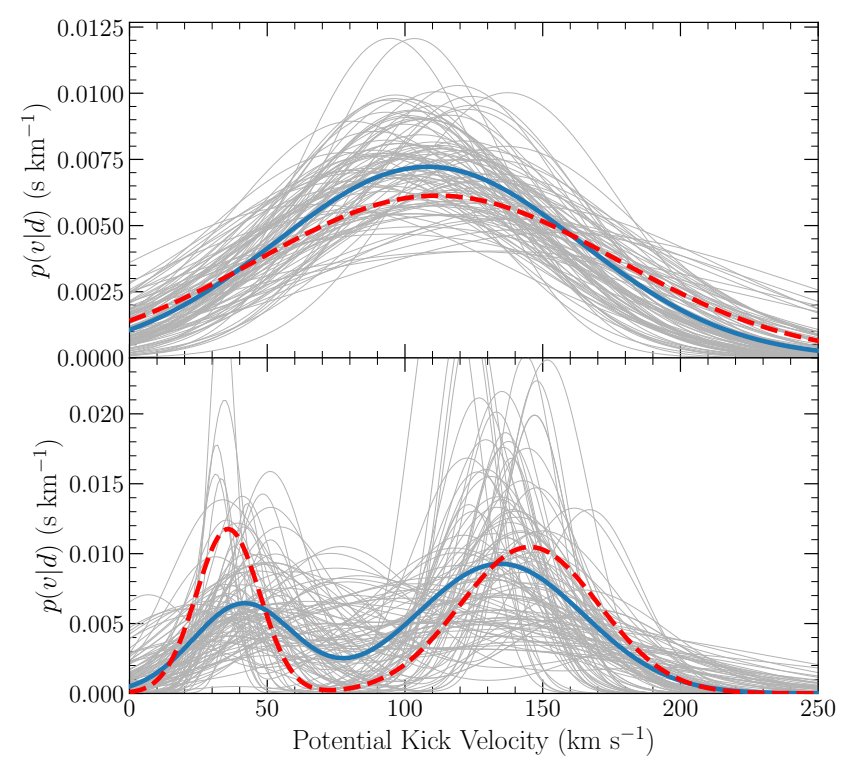

Figure 7. Realisations of the inferred unimodal (top) and bimodal (bottom) distributions for potential kick velocities (v), inferred from the data $(\mathrm{d})$. The blue lines represent the model corresponding to the median values from the posterior sample for data from all the systems in the sample, while the red dashed lines represent the model based on the median from the posterior sample for data from the 12 systems with systemic radial velocity $(\gamma)$ constraints. The faint gray lines are a small random subset from the posterior MCMC sample for all the systems, as a demonstration of uncertainty.

the Eddington luminosity (Maccarone 2003; Kalemci et al. $2013)$ to estimate a distance of $1.7 \mathrm{kpc}$. We have run our simulations using the distance inferred from the Gaia parallax and also using the distance limits as mentioned above. As this distance is better constrained than the Gaia DR-2 distance, we have used the former for estimating the PKV distribution of MAXI J1820+070.

\section{DISCUSSION}

\subsection{Potential kick velocity distribution - BHXB population}

We investigated the distribution of the potential kick velocities in BHXB systems based on the results in Section 6 . Given the probabilistic nature of these results (probability distributions for the predicted velocity of each system), we follow the Bayesian hierarchical methodology outlined by Mandel (2010) and Hogg et al. (2010) for fitting a distribution model. We evaluate the distribution of posteriors with unimodal (with mean of $\mu$ and standard deviation of $\sigma$ ) and bimodal (with means of $\mu_{1}, \mu_{2}$, standard deviations of $\sigma_{1}, \sigma_{2}$, and weights of $w_{1}$ and $w_{2}=1-w_{1}$ ) gaussian models. For this purpose, we used a Hamiltonian Markov chain Monte Carlo (MCMC) algorithm (Neal 2012; Betancourt 2017) with No U-Turn Sampling (NUTS; Hoffman \& Gelman 2011) as implemented in PyMC3 (Salvatier et al. 2016). We assumed uninformative priors for all parameters 
Table 4. Potential kick velocity (PKV) distributions for systems that have a systemic radial velocity measurement $(\gamma)$ in the literature. The measured quantities are the various parameters for the BHXB systems that were available in the literature, and the estimated quantities are the values that have been determined in this work using the MC simulations code described in Section. $\mathrm{d}_{\text {lit }}$ is the best distance estimate available in the literature for some of these systems. The Gaia offset corrected parallax measurement (as described in Section 5.1) and the distance distribution $\left(\mathrm{d}_{\text {post }}\right)$ estimated using the LMXB Milky way density prior are reported. The $5^{t h}$ and $95^{t h}$ percentiles of these distributions are the upper and lower limits on the distance estimated using the Milky Way prior. For distances in the literature that were just a range, a uniform distribution was used as the input to the MC code. For the distances in literature that have been reported with error bars, the input to our MC code was a Gaussian distribution. The PKV is reported as the median of the PKV probability distribution with the lower and upper limits representing the $5^{\text {th }}$ and $95^{\text {th }}$ percentiles. $\mathrm{PKV}_{\text {post }}$ and $\mathrm{PKV}$ lit are the potential kick velocity distributions using the $d_{\text {post }}$ and the $d_{l i t}$ as input distance distributions, respectively. The last column is the suggested birth pathway for the BH in each system and could be SN (Supernova), DC (Direct collapse) or U (Unsure). Refer to Section 7.1 and Section 7.4 for the explanation of the suggested birth pathway. References: [1] Gaia Collaboration et al. (2018); [2] González Hernández \& Casares (2010); [3] Cantrell et al. (2010); [4] González Hernández et al. (2008); [5] Gelino et al. (2006); [6] Orosz et al. (1996); [7] Hynes (2005); [8] Casares et al. (2004); [9] Shahbaz et al. (1999); [10] Hjellming \& Rupen (1995); [11] Orosz et al. (2001); [12] MacDonald et al. (2014); [13] Gies et al. (2008); [14] Miller-Jones et al. (2009b); [15] Casares \& Charles (1994); [16] Orosz et al. (1998); [17] Heida et al. (2017); [18] Russell et al. (2014); [19] Reid et al. (2014); [20] Steeghs et al. (2013); [21] This work.

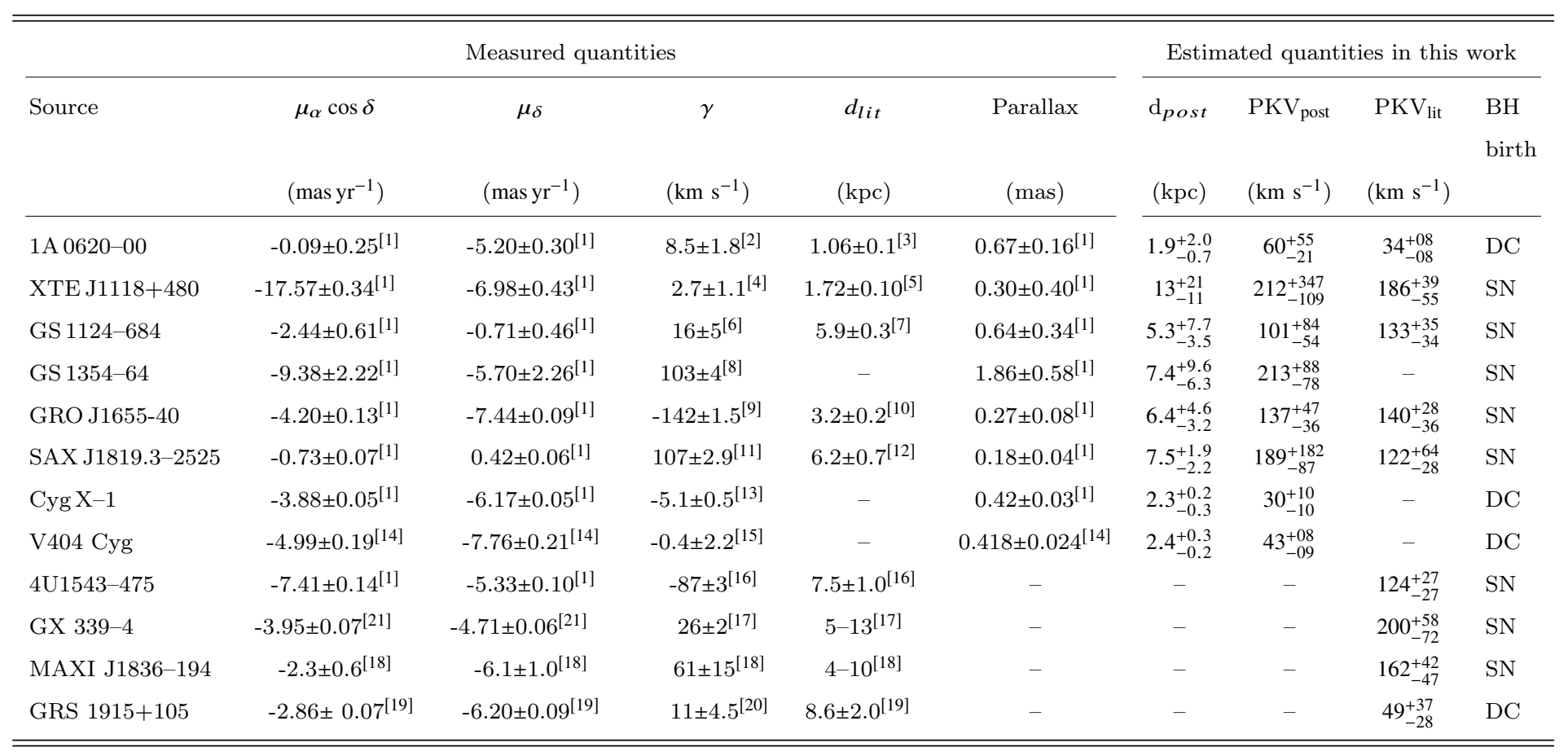

in the models and verified convergence in the final samples for all parameters using the Gelman-Rubin diagnostic test (Gelman \& Rubin 1992), with $\hat{R}<1.001$ for all parameters $(\hat{R}$ closer to one indicates certainty of convergence of the chains).

We evaluated the distribution with both models over two sets of velocities, with one set containing all the BHXB systems in our sample (16 systems), and a second set containing only the systems with systemic radial velocities that have been measured in the literature ( 12 systems). The results for both models in both cases are tabulated in Table 6 and plotted in Fig. 7.

Due to the low number of "events" (number of systems for which we have kick velocity PDFs), we used the corrected Akaike information criterion (AICc; Akaike 1974; Cavanaugh 1997; Burnham \& Anderson 2002) to compare the models. As tabulated in Table 6, the unimodal distribution is clearly favoured in both cases (a smaller AICc suggests a better model). Removal of systems with loose systemic radial velocity constraints provides a marginal relative improvement to the bimodal model, as the difference between the AICc parameter reduced when evaluated for a sample devoid of loose systemic radial velocity constraints as compared to a sample that consisted of all 16 systems. It is worth noting however, that the clear separation of posterior constraints for $\mu_{1}$ and $\mu_{2}$ in the bimodal model (given the uninformative priors) hints at the possibility of a bimodal nature for the distribution of the potential BHXB kicks, but testing this model is currently hampered by the low number of BHXB systems with potential kick velocity constraints.

To demonstrate that neither of the two components in the bimodal model are caused by a single source, we plot all individual distributions in Figure 8. We find that there are at least four systems (first four systems in Figure 8) contributing to the lower velocity peak $\left(41 \mathrm{~km} \mathrm{~s}^{-1}\right)$, which could be consistent with birth of the BH by direct collapse. We find at least nine systems (SAX J1819-2525 to Swift J1753.5-0127 in Figure 8) clearly contributing to 
Table 5. Potential kick velocity (PKV) distributions for systems that do not have a systemic radial velocity measurement $(\gamma)$ in the literature. For these systems, we calculated the predicted systemic radial velocity $(\bar{\gamma})$ for pure Galactic rotation at at a projected distance on the Galactic plane (see Section 5.2 for more details). We run the MC simulations code for five possible systemic radial velocity distributions. The measured quantities are the various parameters for the BHXB systems that were available in the literature, and the estimated quantities are the values that have been determined in this work using the MC simulations code described in Section 6 . $\mathrm{d}_{\text {lit }}$ is the best distance estimate available in the literature for some of these systems. The Gaia offset corrected parallax measurement (as described in Section 5.1) and the distance distribution $\left(\mathrm{d}_{\text {post }}\right)$ estimated using the LMXB Milky Way density prior are reported. The $5^{t h}$ and $95^{t h}$ percentiles of these distributions are the upper and lower limits on the distance estimated using the Milky Way prior. For distances in the literature that were just a range, a uniform distribution was used as the input to the MC code. For the distances in literature that have been reported with error bars, the input to our MC code was a Gaussian distribution. The PKV is reported as the median of the PKV probability distribution with the lower and upper limits representing the $5^{t h}$ and $95^{t h}$ percentiles. PKV post and $P K V_{l i t}$ are the potential kick velocity distributions using the $d_{\text {post }}$ and the $d_{\text {lit }}$ as input distance distributions, respectively. The last column is the suggested birth pathway for the BH in each system and could be SN (Supernova), DC (Direct collapse) or U (Unsure). We have stated that just for the central predicted systemic radial velocity. Refer to Section 7.1 and Section 7.4 for the explanation of the suggested birth pathway. References: [1] This work; [2] della Valle et al. (1994); [3] Cadolle Bel et al. (2007) [4] Gaia Collaboration et al. (2018); [5] Kirsten et al. (2014)

\begin{tabular}{|c|c|c|c|c|c|c|c|c|c|}
\hline \multicolumn{5}{|c|}{ Measured quantities } & \multicolumn{5}{|c|}{ Estimated quantities in this work } \\
\hline Source & $\begin{array}{c}\mu_{\alpha} \cos \delta \\
\left(\mathrm{mas} \mathrm{yr}^{-1}\right)\end{array}$ & $\begin{array}{c}\mu_{\delta} \\
\left(\operatorname{mas~yr}^{-1}\right)\end{array}$ & $\begin{array}{c}d_{l i t} \\
(\mathrm{kpc})\end{array}$ & $\begin{array}{l}\text { Parallax } \\
\text { (mas) }\end{array}$ & $\begin{array}{c}\mathrm{d}_{\text {post }} \\
(\mathrm{kpc})\end{array}$ & $\begin{array}{c}\bar{\gamma} \\
\left(\mathrm{km} \mathrm{s}^{-1}\right)\end{array}$ & $\begin{array}{l}\mathrm{PKV}_{\text {post }} \\
\left(\mathrm{km} \mathrm{s}^{-1}\right)\end{array}$ & $\begin{array}{c}\mathrm{PKV}_{\text {lit }} \\
\left(\mathrm{km} \mathrm{s}^{-1}\right)\end{array}$ & $\mathrm{BH}$ birth \\
\hline \multirow[t]{5}{*}{ GRS 1716-249 } & $-1.7 \pm 1.25^{[1]}$ & $-2.48 \pm 0.75^{[1]}$ & $2.4 \pm 0.4^{[2]}$ & - & - & $-110 \pm 50$ & - & $99_{-45}^{+68}$ & \\
\hline & & & & & & $-60 \pm 50$ & - & $75_{-31}^{+54}$ & \\
\hline & & & & & & $-10 \pm 50$ & - & $67_{-27}^{+41}$ & $\mathrm{U}$ \\
\hline & & & & & & $40 \pm 50$ & - & $75_{-32}^{+54}$ & \\
\hline & & & & & & $90 \pm 50$ & - & $100_{-47}^{+68}$ & \\
\hline \multirow[t]{5}{*}{ Swift J1753.5-0127 } & $0.84 \pm 0.06^{[1]}$ & $-3.64 \pm 0.06^{[1]}$ & $6 \pm 2^{[3]}$ & $0.02 \pm 0.13^{[4]}$ & $8.8_{-4.0}^{+12}$ & $-7 \pm 50$ & $212_{-104}^{+114}$ & $154_{-84}^{+116}$ & \\
\hline & & & & & & $43 \pm 50$ & $196_{-89}^{+118}$ & $142_{-66}^{+101}$ & \\
\hline & & & & & & $93 \pm 50$ & $240_{-115}^{+128}$ & $\begin{array}{r}177_{-103}^{+135}\end{array}$ & $\mathrm{SN}$ \\
\hline & & & & & & $143 \pm 50$ & $192_{-82}^{+118}$ & $153_{-63}^{+88}$ & \\
\hline & & & & & & $193 \pm 50$ & $204_{-77}^{+115}$ & $173_{-65}^{+86}$ & \\
\hline \multirow[t]{5}{*}{ MAXI J1820+070 } & $-3.41 \pm 0.19^{[4]}$ & $-5.90 \pm 0.22^{[4]}$ & $1.7-3.9^{[1]}$ & $0.31 \pm 0.11^{[4]}$ & $4.4_{-2.0}^{+5.1}$ & $-67 \pm 50$ & $185_{-83}^{+147}$ & $153_{-77}^{+128}$ & \\
\hline & & & & & & $-17 \pm 50$ & $143_{-63}^{+104}$ & $110_{-49}^{+97}$ & \\
\hline & & & & & & $33 \pm 50$ & $111_{-41}^{+79}$ & $86_{-30}^{+67}$ & $\mathrm{SN}$ \\
\hline & & & & & & $83 \pm 50$ & $103_{-33}^{+62}$ & $84_{-27}^{+50}$ & \\
\hline & & & & & & $133 \pm 50$ & $109_{-33}^{+62}$ & $\begin{array}{r}98_{-34}^{+75} \\
\end{array}$ & \\
\hline \multirow[t]{5}{*}{ VLA J2130+12 } & $-0.07 \pm 0.13^{[5]}$ & $-1.26 \pm 0.29^{[5]}$ & - & $0.45 \pm 0.08^{[5]}$ & $2.4_{-1.8}^{+3.4}$ & $-90 \pm 50$ & $146_{-74}^{+116}$ & - & \\
\hline & & & & & & $-40 \pm 50$ & $101_{-47}^{+95}$ & - & \\
\hline & & & & & & $10 \pm 50$ & $82_{-32}^{+63}$ & - & $\mathrm{U}$ \\
\hline & & & & & & $60 \pm 50$ & $86_{-34}^{+60}$ & - & \\
\hline & & & & & & $110 \pm 50$ & $111_{-49}^{+79}$ & - & \\
\hline
\end{tabular}

the higher velocity peak $\left(136 \mathrm{~km} \mathrm{~s}^{-1}\right)$. Better constraints on some of the measured parameters for systems like GS 1124684, VLA J2130+12, GS 1352-64, Swift J1753.5-0127 and SAX J1819-2525 might help in tightening their PKV probability distributions, which could change the form (and interpretation) of the PKV distribution of BHXB population. With the current available data on BHXBs, our results provide the best constraint we can obtain for the PKV distribution of BHXBs.

\subsection{Potential Kick velocity interpretation}

The potential kick velocity (PKV) that we estimate here is not the actual natal kick the $\mathrm{BH}$ receives when it is born, but is the potential peculiar velocity of the system, with which it may have been kicked out of the Galactic plane. Calculating the actual $\mathrm{BH}$ natal kick from the BHXB peculiar velocity at Galactic plane crossing is complex and depends on modelling the system (Repetto et al. 2012, 2017), taking into account the orbital period, the component masses of the binary sys- 
Table 6. Model estimates for the potential kick velocity distribution using unimodal and bimodal gaussian models when all systems are considered and when only systems with well constrained radial systemic velocity $(\gamma)$ are considered. Reported values are medians $(\mu)$ of the posterior distributions, along with uncertainties $(\sigma)$ and all values are in $\mathrm{km} \mathrm{s}^{-1} . w_{1}$ is the weight of the Gaussian distribution with the mean $\mu_{1}$. AICc is the corrected Akaike information criterion. A smaller AICc suggests that the model is more favourable.

\begin{tabular}{lccccccl}
\hline \hline & & $\mu_{1}$ & $\sigma_{1}$ & $\mu_{2}$ & $\sigma_{2}$ & $w_{1}$ & AICc \\
\hline \multirow{2}{*}{ All systems } & Unimodal & $107 \pm 16$ & $56 \pm 14$ & - & - & - & -16.3 \\
& Bimodal & $41 \pm 14$ & $19 \pm 10$ & $136 \pm 17$ & $32 \pm 18$ & $0.3 \pm 0.1$ & -2.2 \\
\hline \multirow{2}{*}{ Systems with $\gamma$} & Unimodal & $112 \pm 22$ & $65 \pm 19$ & - & - & - & -2.4 \\
& Bimodal & $35 \pm 10$ & $11 \pm 9$ & $145 \pm 15$ & $30 \pm 17$ & $0.3 \pm 0.1$ & 10.7 \\
\hline
\end{tabular}

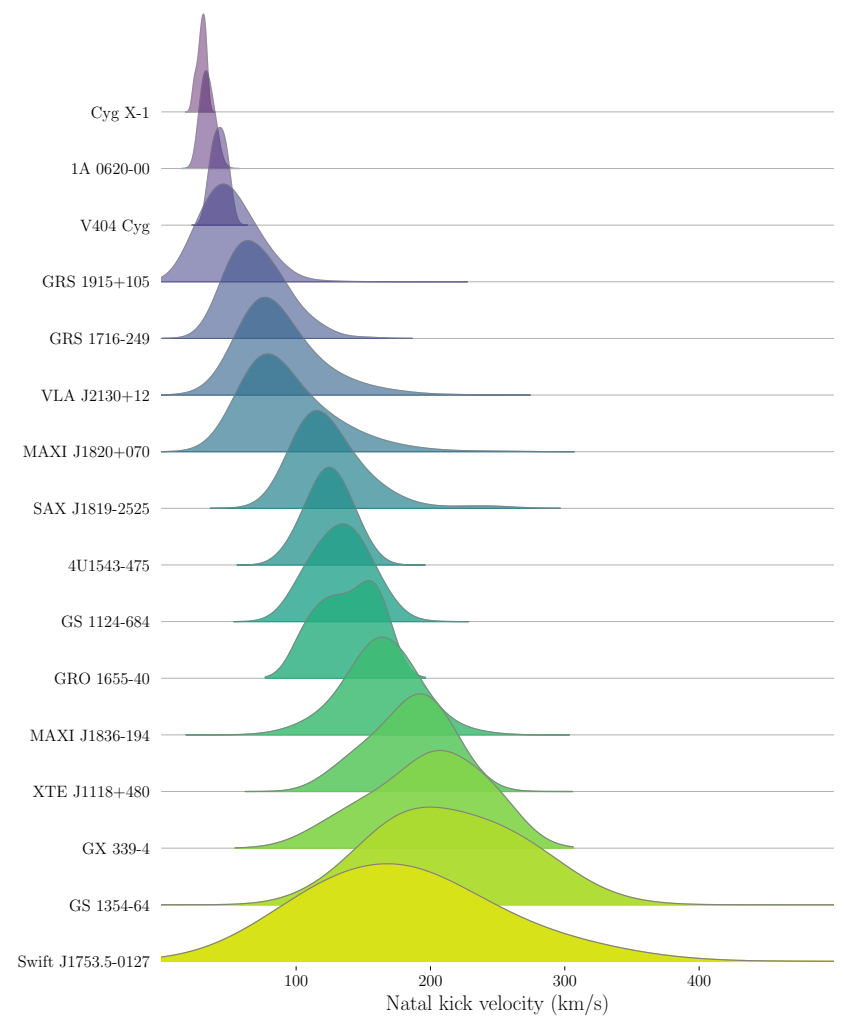

Figure 8. PKV probability distributions of the 16 BHXBs in our sample. It can be seen that no single system is responsible for either of the two most prominent peaks in the aggregated natal kick distribution.

tem, the direction of the kick and the mass ejected in the SN explosion (Nelemans et al. 1999). It also involves simulating the subsequent dynamical interaction of the sources and evolution of the binary system. These parameters are currently not well constrained for most systems, and thus simulating the evolution of these systems to determine the actual $\mathrm{BH}$ natal kick is out of the scope of this paper. Hence the peculiar velocity of the system at Galactic plane crossing is currently the best proxy that we can use for BH natal kicks in a population-wide analysis such as this one. Since most star forming regions are in the Galactic plane, it is probable that most BHXBs form in the Galactic plane. Since we do not know the time of $\mathrm{BH}$ birth, we instead consider the ve- locity of the system every time it crosses the Galactic plane as a potential velocity kick the system might have received.

According to Mignard (2000), stellar velocity dispersions due to Galactic interactions are the order of $\sim 50 \mathrm{~km} \mathrm{~s}^{-1}$ for old systems (and lower for younger systems). Since BHXBs are more massive than these systems, they will suffer even lower velocity dispersions. Thus we classify systems with potential kick velocities higher than the $\sim 50 \mathrm{~km} \mathrm{~s}^{-1}$ limit as systems that might have received strong kicks, and hence the BH in the BHXB might have formed in a SN explosion. We suggest that the systems with a median PKV of less than $\sim 50 \mathrm{~km} \mathrm{~s}^{-1}$ are systems with weak potential kicks, and thus could well have formed by direct collapse rather than a SN explosion. Systems that have a PKV distribution such that their median is $>50 \mathrm{~km} \mathrm{~s}^{-1}$ but the $5^{\text {th }}$ is lower than $50 \mathrm{~km} \mathrm{~s}^{-1}$ do not clearly fall into either category, and hence we could not deduce their likely birth mechanism. We find two such sources in our sample, namely VLA J2130+12 and GRS 1716-249. Ten systems in our sample are likely to have been formed in a SN explosion, whereas four systems could plausibly have formed by direct collapse. We summarise the results of our analysis in Tables 4 and 5 .

The PKV probability distribution of GRS 1915+105 (median $\sim 49 \mathrm{kms}^{-1}$ ) suggests that it could have been born via direct collapse, as the PKV is within the velocity dispersion limits for Galactic scattering $\left(\sim 50 \mathrm{~km} \mathrm{~s}^{-1}\right.$ for late type stellar systems; Mignard 2000). According to our results the $\mathrm{v}_{95}$ values for V404 Cyg and 1A 0620-00 are lower than the v $_{95}$ for GRS $1915+105$, suggesting that V404 Cyg and 1A 0620-00 might also have been born without a strong natal kick. The probability of a compact object receiving a kick between angle $\theta$ and $\theta+d \theta$ during a SN explosion varies as $\frac{1}{2} \sin \theta d \theta$, where $\theta$ is the angle the kick makes with the direction of the peculiar velocity of the system before the explosion. Thus it is also possible that these low PKV systems were born with a SN explosion but their velocity was reduced due to an asymmetry in the explosion counteracting the recoil kick. For Cyg X-1, the median of the PKV we obtain is in agreement with the estimation of Wong et al. (2012) and suggests a direct collapse birth (albeit with the caveat that this is a high mass X-ray binary and has never crossed the Galactic plane in its lifetime, so our methodology is not strictly applicable).

For our interpretation of PKV as the BH natal kick, we are assuming that none of the systems in our sample were born in GCs. There have been no confirmed BHXBs in GCs, though there are some candidates (Strader et al. 2012; Camilo et al. 2000; Chomiuk et al. 2013; Miller-Jones 


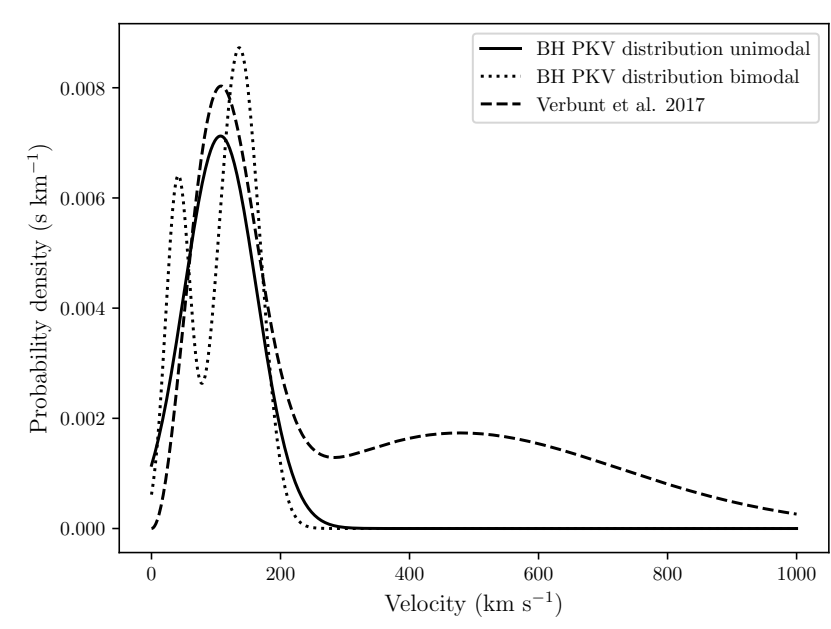

Figure 9. Comparison of best fit unimodal and bimodal BHXB PKV distributions to the best pulsar kick velocity distribution. The BHXB PKV unimodal distribution is represented by the solid line and has a median of $107 \mathrm{~km} \mathrm{~s}^{-1}$. The BHXB PKV bimodal distribution is represented by the dotted line. The best fit, two Maxwellian model for the peculiar velocities of pulsars (Verbunt et al. 2017) is denoted by the dashed line. The unimodal BHXB PKV distribution median is close to the low kick pulsar kick velocity component. There is a dearth of extremely high PKV BHs corresponding to the high velocity Maxwellian component for the pulsar velocity distribution.

et al. 2015). GCs only contain $5-10 \%$ of the total number of known Galactic X-Ray binaries (Clark 1975; Bahramian et al. 2014), so this population is likely to be a small fraction of the total BHXBs in the Galaxy.

The Galactocentric orbits in the past 10 Gyrs also show that only four (GRO 1655-40, SAX J1819-2525, GX 339-4 and MAXI J1836-194) out of the 16 systems ever get within $2 \mathrm{kpc}$ of the Galactic centre, which is the truncation radius for a spheroidal bulge model (Dehnen \& Binney 1998). We note that we have classified these systems as having a SN birth, but due to their possible association with being bulge objects we cannot rule out the high PKV as being due to bulge formation and/or interactions.

\subsection{Comparison with NS natal kicks}

The kick velocity distribution of pulsars has been extensively studied (e.g. Hobbs et al. 2005; Fryer et al. 1998; Arzoumanian et al. 2002), whereas there is no well documented kick velocity measurement catalogue for neutron star X-ray binaries (NS XRBs). Thus to understand the relation between NS and BH natal kicks, we compare our BHXB PKV distribution with the pulsar kick velocity distribution (Verbunt et al. 2017). BHXBs and pulsars suffer from different observational biases. The distance to pulsars is usually smaller than the distance to BHXBs, and thus their detection is less affected by extinction. Pulsars just have their two dimensional velocities measured, there are no systemic velocity measurements for pulsars. Also, it is possible to observe pulsars that might have received strong enough kicks to disrupt the binary they were a part of, whereas our data sample only consists of BHs that are in binaries. Keeping these biases in mind, we compare BHXB and pulsar kick velocities.

Theory suggests that fallback of matter ejected in a SN onto the proto-neutron star can give rise to BHs, so we expect similarities in the kick distributions of BHs and NSs. The most recent NS peculiar velocity distribution is fit better with two Maxwellian components than one (V17). Our bimodal Gaussian (Figure 9) fit to the data shows that the medians of the two Gaussians in the BHXB PKV distribution $\left(41 \pm 14 \mathrm{~km} \mathrm{~s}^{-1}\right.$ and $\left.136 \pm 17 \mathrm{~km} \mathrm{~s}^{-1}\right)$ are lower than the NS peculiar velocity peaks $\left(120 \mathrm{~km} \mathrm{~s}^{-1}\right.$ and $\left.540 \mathrm{~km} \mathrm{~s}^{-1}\right)$ for the best fit model by V17 by a factor of 3-4. This may suggest that $\mathrm{BHs}$ receive weaker natal kicks as compared to NSs by a factor that is similar to the mass ratios of standard BHs and NSs. This is in contrast to the conclusion of Repetto et al. (2012), where it was shown that BHs and NSs get equally strong kicks. This mismatch of the medians of the two components of the BHXB PKV distribution and pulsar kick velocity distribution could also be because of different kick mechanisms in BHs and NSs. For BHs the low velocity kick is probably due to formation by direct collapse, whereas low NS kicks have been attributed to various mechanisms like ultra-stripped SNe (Tauris et al. 2015), electron capture SNe (Gessner \& Janka 2018) and collapse of low iron mass cores (Podsiadlowski et al. 2004).

Our unimodal fit to the BHXB PKV data matches the lower maxwellian component of the pulsar kick velocity distribution (Figure 9). However, we are biased against stronger kicks, since they can unbind BHXBs and make them unobservable whereas pulsars can be observed even in an unbound state. Due to this selection bias, we expected the BHXB PKV distribution kicks to be lower than the V17 peculiar velocities.

\subsection{Supernova mass loss}

We find that there are at least nine systems of the 16 in our sample set that clearly received strong natal kicks $\left(>50 \mathrm{~km} \mathrm{~s}^{-1}\right)$. This makes SN birth more probable for these systems than direct collapse $\mathrm{BH}$ birth. We note that our sample set may be biased against observing direct collapse BHs. This is because they will not receive strong kicks and will be located close to the Galactic plane, where detecting distant BHXBs is challenging due to high extinction and scattering.

Symmetric mass loss $(\Delta M)$ during a $\mathrm{SN}$ explosion is one of the reasons for a natal kick. However, this mass loss has to be $<0.5\left(M_{\mathrm{He}}+m\right)$ for the binary to remain bound (Blaauw 1961), where $M_{\mathrm{He}}$ is the mass of the progenitor helium star and $\mathrm{m}$ is the mass of the donor star. Thus the maximum possible recoil velocity due to symmetric mass loss can be estimated by constraining the maximum possible mass ejected in the BHXB system without unbinding the binary. This was given by Nelemans et al. (1999) as

$$
v_{\text {sys }}=213\left(\frac{\Delta M}{\mathrm{M}_{\odot}}\right)\left(\frac{m}{\mathrm{M}_{\odot}}\right)\left(\frac{P_{\mathrm{re}-\text { circ }}}{\text { days }}\right)^{-1 / 3}\left(\frac{\left(M_{\mathrm{BH}}+m\right)}{\mathrm{M}_{\odot}}\right)^{-5 / 3} \mathrm{~km} \mathrm{~s}^{-1}
$$

where $\Delta \mathrm{M}, \mathrm{M}_{\mathrm{BH}}$ and $\mathrm{m}$ are the mass ejected in the $\mathrm{SN}$, mass of the $\mathrm{BH}$ and mass of the donor immediately after the $\mathrm{SN}$, respectively. $\mathrm{P}_{\mathrm{re}-\text { circ }}$ is the period of the re- 
circularised orbit of the system after the formation of the $\mathrm{BH}$, and it is assumed that no mass transfer occurs until the re-circularisation of the orbit is complete. $\mathrm{v}_{\text {sys }}$ is the recoil kick velocity the system should have received due to the symmetric mass loss in the SN explosion. Nelemans et al. (1999) estimated the maximum possible recoil kick velocities for GS 1124-684 and GRO 1655-40 and found that they were limited to $<40 \mathrm{~km} \mathrm{~s}^{-1}$. Our estimated PKV for all these systems is greater than those estimated by Nelemans et al. (1999) in the case of only symmetric mass ejection. We thus suggest that the observed kick velocity of these systems is not only because of symmetric mass loss in a SN explosion, but due to these systems receiving additional acceleration due to asymmetries associated with the SN explosion (Janka 2017).

Gualandris et al. (2005) suggested that XTE J1118+480 received an asymmetric kick that put the system in its current Galactocentric orbit. We measured a PKV of $186 \pm 28 \mathrm{~km} \mathrm{~s}^{-1}$ for XTE J1118+480, which agrees with the peculiar velocity measurement of Gualandris et al. (2005). Systems like 4U1543-475, GS 1354-64, SAX J1819.3-2525 and MAXI J1820+070 also have high enough PKVs to suggest asymmetries in the $\mathrm{SN}$ explosions, keeping in mind that we are assuming that these systems are similar to the ones already studied in the literature (e.g. Nelemans et al. 1999; Gualandris et al. 2005). The observed metal abundances in XTE J1118+480 (González Hernández et al. 2006), GRO J1655-40 (González Hernández et al. 2008) and SAX J1819.3-2525 (Orosz et al. 2001) also point towards contamination of these sources by a $\mathrm{SN}$ and support the hypothesis that they were probably born in SN explosions.

\subsection{Natal kicks and z-distribution}

In the past, the distribution of the distance from the Galactic plane $(|z|)$ has been used to determine the strength of BH natal kicks (e.g. van Paradijs \& White 1995; Jonker \& Nelemans 2004; Repetto et al. 2017). We plot the median of the current height probability distributions of all BHXBs in our sample using our distance posterior distributions (Table 7) in Figure 10. It can be seen that all systems with a potential kick velocity $<100 \mathrm{kms}^{-1}$ are within $500 \mathrm{pc}$ of the Galactic plane, other than VLA J2130+12. We conducted Pearson and Spearman rank correlation tests to check the correlation of the distance to the Galactic plane $(|z|)$ with PKVs of BHXBs. We found a potential correlation between the two, with correlation coefficients of $0.51 \pm 0.15(\mathrm{p}=0.02)$ and $0.56 \pm 0.13(\mathrm{p}=0.01)$ for Pearson and Spearman Rank tests respectively. This, however does not suggest that systems at small heights from the Galactic plane received low kick velocities because of the two outliers GRO J1655-40 and GS 1354-64. This could be because the systems are passing through the Plane at the present time, which is the case for GRO J1655-40 as it is on a path towards the Galactic plane in its orbit (Gandhi et al. 2019). The distance estimate of GS 1354-64 is poorly constrained and could be the reason for a misleading current height above the Galactic plane. Thus, the strength of PKV a BHXB receives cannot be determined solely by the current $|z|$. Hence, the approach that we take is currently the best that can be done to estimate natal kick distributions.

Comparisons between the root mean square distance from the Galactic plane $\left(z_{r m s}\right)$ for BHXBs and NSs have led to contrasting claims about BH natal kicks. NSs and $\mathrm{BHs}$ have different observational biases and these contrasting claims could be an artefact of that. White \& van Paradijs (1996) suggested that BHs get reduced kicks as compared to NSs. Jonker \& Nelemans (2004) updated the distances to these systems and showed that $\mathrm{BH}$ natal kicks are as strong as those of NSs. We now update the $z_{r m s}$ as we have new distances (in some cases better constrained distances) for BHXBs. Our analysis gives the $z_{r m s}$ of BHXBs as $\sim 700 \mathrm{pc}$, which is higher than those estimated by White \& van Paradijs (1996) ( 410 pc) and Jonker \& Nelemans (2004) ( 625 pc), but lower than the most recent estimate by Repetto et al. (2017) ( 980 pc). If we exclude the halo object XTEJ1118+480, the $z_{r m s}$ for BHXBs drops down to $595 \mathrm{pc}$. If we are to believe the relation between $z_{r m s}$ and BHXB kick distribution, this would then suggest that NSXBs $\left(z_{r m s}=1 \mathrm{kpc}\right.$ (van Paradijs \& White 1995)) receive stronger kicks as compared to BHXBs. We arrived at a similar conclusion when we compared pulsar kick velocities with BHXB PKVs in Section 7.3, but with the caveat of the selection bias of our sample of BHXBs. Hence, even though the current $|z|$ of the BHXBs in our data sample is not a strong proxy for the PKV of individual systems, $z_{r m s}$ is a good way to compare the PKV distributions of our sample of BHXBs to the NSs. This could be because systems spend most of their time close to the orbit extrema, and when we average the current heights of a sample, we remove the bias of the epoch of observation.

\subsection{Natal kicks and black hole mass}

Attempts have been made to understand if there is any relation between $\mathrm{BH}$ mass and the natal kick it receives (Mirabel 2017). This could assist in understanding the correlation between the final pathway a progenitor takes to become a $\mathrm{BH}$ and the mass of the $\mathrm{BH}$. The current theoretical understanding does not show a direct correlation and suggests that there is no clean mass cut off for direct collapse formation (Sukhbold et al. 2016). We have obtained observational constraints on the potential kicks of 16 BHXBs (Figure 10), which increases the sample size and updates the estimations of BHXB kicks by Mirabel (2017) and Gandhi et al. (2019) (current peculiar velocity used as proxy in both works). We conducted correlation tests, which indicated negligible correlation between $\mathrm{BH}$ mass and kick, with a Pearson Rank coefficient of $-0.23 \pm 0.17(\mathrm{p}=0.21)$ and a Spearman Rank coefficient of $-0.2 \pm 0.17(\mathrm{p}=0.40)$. We used a standard mass of $8 \pm 3 \mathrm{M}_{\odot}$ (Kreidberg et al. 2012) for the systems that do not have a dynamically measured mass (see Table 7 for a summary of masses used). Removing these systems from the test sample gave Pearson and Spearman rank coefficients of $-0.36 \pm 0.16(p=0.20)$ and $-0.31 \pm 0.17(p=0.28)$, respectively. Using BHXB potential kick velocities as an indicator of $\mathrm{BH}$ natal kick, we suggest that there is no significant dependency of $\mathrm{BH}$ mass on natal kick. This is the first time there has been observational evidence to support the simulations of Sukhbold et al. (2016), although we stress that the large error bars in the masses of the BHs (e.g. Ziółkowski 2008) are a caveat. 

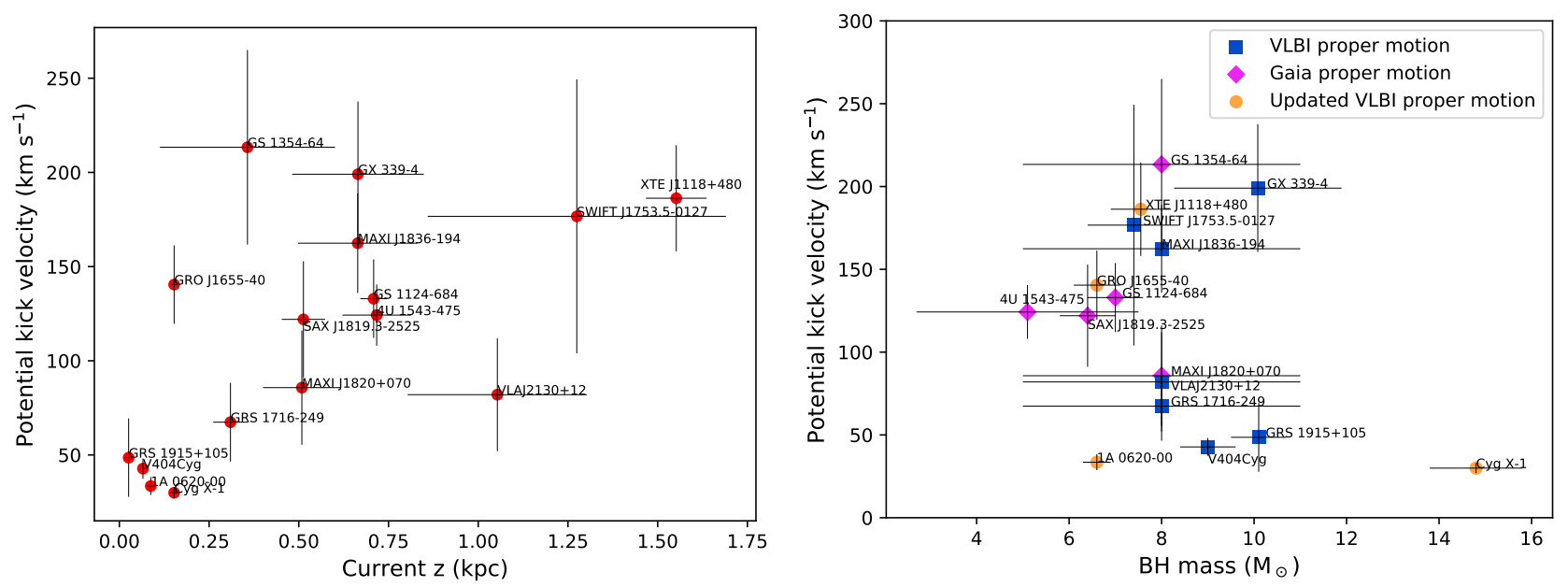

Figure 10. Left: the variation of PKV with the current height of the system above the Galactic plane. There are five systems with a PKV below $100 \mathrm{~km} \mathrm{~s}^{-1}$ are within $500 \mathrm{pc}$ of the Galactic plane. There are two systems that are within $500 \mathrm{pc}$ but have PKVs $>100 \mathrm{~km} \mathrm{~s}$. Right: the variation of PKVs with black hole mass. The systems marked with blue squares have had their proper motion measured by VLBI and the systems marked with pink diamonds had their proper motion measured by Gaia. The systems marked with yellow circles had their proper motions measured by VLBI, but we used the updated proper motions measured by Gaia for our simulations. We have assumed a $\mathrm{BH}$ mass of $8 \mathrm{M}_{\odot}$ to represent the systems that do not have dynamical mass measurements (Kreidberg et al. 2012). The error bars are one standard deviation of the PKV probability distributions. No correlation between BH mass and potential kick velocity is seen, even after disregarding the systems without dynamical mass measurements.

Table 7. Current $|z|$ and PKV estimates for every source, along with the assumed mass used for the correlation test. The PKV is the median of the PKV probability distribution and the error bar is the $1 \sigma$ deviation of the distribution. Current $|z|$ is the median of the current $|z|$ distribution for the estimated distance distributions for each BHXB and the errors are the $1 \sigma$ of the distributions. GS 1354-64, MAXI J1836-194, GRS 1716-249, MAXI J1820+070 and VLA J2130+12 do not have dynamical mass measurements, so we assumed $\mathrm{BH}$ masses of $8 \mathrm{M}_{\odot}$ (Kreidberg et al. 2012).

\begin{tabular}{lcccl}
\hline \hline Source & $\begin{array}{c}\text { PKV } \\
\left(\mathrm{km} \mathrm{s}^{-1}\right)\end{array}$ & $\begin{array}{c}\text { Current }|\mathrm{z}| \\
(\mathrm{kpc})\end{array}$ & $\begin{array}{c}\text { BH mass } \\
\left(\mathrm{M}_{\odot}\right)\end{array}$ & mass Ref \\
\hline 1A 0620-00 & $34 \pm 5$ & $0.09 \pm 0.01$ & $6.6 \pm 0.3$ & van Grunsven et al. (2017) \\
XTE J1118+480 & $186 \pm 28$ & $1.55 \pm 0.09$ & $7.55 \pm 0.65$ & Khargharia et al. (2013) \\
GS 1124-684 & $133 \pm 21$ & $0.71 \pm 0.04$ & $7 \pm 0.6$ & Casares (2007) \\
GS 1354-64 (BW Cir) & $213 \pm 52$ & $0.36 \pm 0.24$ & - & - \\
4U 1543-475 & $124 \pm 16$ & $0.72 \pm 0.1$ & $2.7-7.5$ & Orosz et al. (1998) \\
GRO J1655-40 & $140 \pm 21$ & $0.15 \pm 0.01$ & $6.6 \pm 0.5$ & Casares (2007) \\
GX 339-4 & $199 \pm 39$ & $0.66 \pm 0.18$ & $8.28-11.89$ & Sreehari et al. (2018) \\
GRS 1716-249 & $67 \pm 21$ & $0.31 \pm 0.05$ & - & Masetti et al. (1996) \\
Swift J1753.5-0127 & $177 \pm 73$ & $1.3 \pm 0.42$ & $>7.4 \pm 1.2$ & Shaw et al. (2016) \\
SAX J1819.3-2525 & $122 \pm 31$ & $0.51 \pm 0.06$ & $6.4 \pm 0.6$ & MacDonald et al. (2014) \\
MAXI J1820+070 & $86 \pm 30$ & $0.51 \pm 0.11$ & - & - \\
MAXI J1836-194 & $162 \pm 27$ & $0.66 \pm 0.17$ & - & Russell et al. (2014) \\
GRS 1915+105 & $49 \pm 21$ & $0.03 \pm 0.01$ & $10.1 \pm 0.6$ & Steeghs et al. (2013) \\
Cyg X-1 & $30 \pm 3$ & $0.15 \pm 0.01$ & $14.8 \pm 1.0$ & Orosz et al. (2011) \\
V404 Cyg & $43 \pm 5$ & $0.07 \pm 0.01$ & $9 \pm 0.6$ & Khargharia et al. (2010) \\
VLA J2130+12 & $82 \pm 30$ & $1.05 \pm 0.25$ & - & - \\
\hline
\end{tabular}

\subsection{BHs in Globular clusters}

XTE J1118+480 is argued to be a GC escapee because of its high peculiar velocity and high Galactic latitude. We find at least five systems with PKVs comparable to XTE J1118+480, making this system non-unique. However, most LMXBs are relatively old systems and thus may have come from low metallicity environments like GCs. So a GC origin of these high PKV systems cannot be ruled out. Since the majority of the systems in our sample get strong kicks, then if this distribution is characteristic of BHs it would imply that the retention fraction may be fairly low in GCs. As globular clusters (GC) are dense stellar environments, they are ideal sites for forming binaries. The escape velocities of GCs are on the order of a few tens of $\mathrm{km} \mathrm{s}^{-1}$ (Gnedin et al. 2002; Belczynski et al. 2006). Thus, most of the BHs should be eventually ejected from the GC due to kicks associated with their birth or dynamical interactions (Kulkarni 
et al. 1993; Drukier 1996). The recent increase in the number of BHXB candidates detected in GCs challenges this theory (Strader et al. 2012; Camilo et al. 2000; Chomiuk et al. 2013; Miller-Jones et al. 2015; Giesers et al. 2018). Recent simulations have shown that if GCs retain $\sim 1000 \mathrm{BHs}$, then these BHs will produce a considerable population of BHXBs in the GCs (Giesler et al. 2018). Simulations that estimate the BHs retained in GCs use various observationally unconstrained distributions ranging from random flat kick distributions up to some value (Sippel \& Hurley 2013) through to unconstrained retention fractions (Giesler et al. 2018). Our PKV distribution shows that these assumptions should be used with caution. The range of assumptions for the kick distributions also emphasise the need for a consistent, observationally constrained distribution.

According to our results the $20^{\text {th }}$ percentile of the PKV distribution for BHXBs is $\sim 45 \mathrm{~km} \mathrm{~s}^{-1}$, which is the typical $v_{\text {esc }}$ for GCs. This suggests that BHXBs, which could have weaker recoil kicks than isolated BHs due to remaining bound, have a very high probability of getting kicked out from GCs. On the contrary, if BHXBs are Blaauw kick dominated, then isolated BHs have a higher chance of being retained in GCs. Thus, at birth, the retention fraction of BHs in GCs could be lower than the current estimates and may need to be updated. However, we note that GCs are old systems with low metallicity, and we have no observational constraints on how metallicity might affect the kick distribution.

\subsection{Implications for BH-BH systems}

When a star in the binary undergoes a SN explosion, any asymmetry in the kick could misalign the spin of the remnant $\mathrm{BH}$ to the orbital plane. If tidal interactions are then unable to realign the spin, this could potentially give rise to a high degree of misalignment of the spins of the $\mathrm{BH}$ to the orbital plane of the BH-BH binary (Gerosa et al. 2013). Recent observations of the GW merger event GW151226 suggested that natal kicks $>50 \mathrm{~km} \mathrm{~s}^{-1}$ were needed to explain the inferred spin-orbit misalignment of the binary (O'Shaughnessy et al. 2017). The spin measurements for three other GW events GW150914, LVT151012 and GW170104 indicated that there is a possibility for these systems to either have low intrinsic spins or large spins that are misaligned with the binary orbit (Farr et al. 2017). 90\% of our sources have PKVs higher than $50 \mathrm{~km} \mathrm{~s}^{-1}$, thus suggesting that spin-orbit misalignment might be a common phenomenon.

Based on LIGO's BH binary merger observations it was suggested that BHs receive high natal kicks $\sim 200 \mathrm{kms}^{-1}$ if the tidal processes do not realign stellar spins (Wysocki et al. 2018). If however tidal processes do realign the spins, kicks on the order of $50 \mathrm{kms}^{-1}$ are still needed to explain the observations. The bimodality in our natal kick distributions, albeit based on a few sources, could potentially arise from similar tidal effects, though more work needs to done in order to draw definitive conclusions. Theoretical estimates suggest that the majority of $\mathrm{BH}$ binaries that will be observed with the third LIGO run will be part of a population in which the first-born BH is slowly spinning, and support low natal kicks for BHXBs (Bavera et al. 2019; Fuller \& Ma 2019). Measuring PKVs for more BHXBs and future LIGO observations will be essential to reconcile these theoretical expectations of low natal kicks with our observational constraints of high PKVs.

\subsection{BHXB spin-orbit misalignment}

Study of X-ray power density spectra of BHXBs show low frequency quasi periodic oscillations (QPOs) in the power density spectra of almost all BHXB systems (Ingram et al. 2016). Theoretical models suggest that such QPOs could be a result of Lense-Thirring precession (Stella \& Vietri 1998), which is a phenomenon where material out of the $\mathrm{BH}$ equatorial plane (e.g. due to misalignment of the $\mathrm{BH}$ spin and binary orbit) precesses due to relativistic frame dragging. Any natal kick imparted to a $\mathrm{BH}$ at its birth could cause a spin-orbit misalignment in the remnant BHXB. Since realignment timescales of BHXBs are usually longer than the ages of these systems (Martin et al. 2008), realignment of the $\mathrm{BH}$ spin to the orbital plane is not likely to be common (unless the donor star in the BHXB was intially an intermediate mass donor; Fragos \& McClintock 2015). We found that a majority of the BHXBs in our sample set were strongly kicked, which is consistent with the prevalence of low frequency QPOs and provides strong evidence for spinorbit misalignment in BHXBs.

Extensive efforts to measure the spins of BHs in BHXBs have been made in the past decade. The best available methods (García et al. 2018; McClintock et al. 2014) often assume that the spin of the $\mathrm{BH}$ is aligned with the orbital plane of the binary. Our finding that strong natal kicks are imparted to $\sim 85 \%$ of BHXBs suggests that caution may be warranted in assuming the binary orbital inclination when fitting for $\mathrm{BH}$ spin.

\section{CONCLUSIONS}

In this paper, we report on measurements of proper motions of three BHXB systems using VLBI. We collated the proper motions, systemic radial velocities and distances of 16 BHXB systems. We developed a methodology to determine potential kick velocity (PKV) distributions for BHXB systems. We also developed a MC simulation code to account for uncertainties in the measured proper motions, systemic radial velocities and distances when determining the potential kick velocity that the BHXB received, providing robust observational constraints on the possible kick velocities of these systems. We estimated PKV probability distributions for sixteen BHXBs and found that 75\% of our sample has a median potential kick velocity of $>70 \mathrm{kms}^{-1}$, which we interpreted as a majority of BHXBs acquiring strong kicks when they are born. We combined the PKV probability distributions for these 16 BHXBs to obtain an aggregate PKV distribution. We found that a unimodal Gaussian distribution with a mean of $107 \pm 16 \mathrm{~km} \mathrm{~s}^{-1}$ fit the data better than a bimodal distribution, which is potentially consistent with the LIGO's BH-BH merger observations and natal kick estimations. Alternatively, the fit suffers from low number of systems in the data sample and hence we could not rule out a bimodal distribution. We found no significant correlation between PKVs and BH mass. We did not find any strict mass cut off for BHs to form with a $\mathrm{SN}$ or by direct collapse

We conducted Spearman and Pearson rank correlation 
tests to determine the correlation between the current height above the Galactic plane of a BHXB and the potential kick velocity it received. We found that even though there is a potential correlation between the two (coefficient of $\sim 0.5$ for both tests), we should avoid using $|z|$ as a natal kick proxy. We compared our aggregated PKV distribution with the pulsar peculiar velocity distribution (Verbunt et al. 2017) and found that BHs may get weaker kicks than NSs by a factor of 3-4. Our finding that BHXB kick velocities are greater than typical escape velocities of GCs favours a large fraction of BHs being kicked out of GCs. The prevalence of strong kicks in our BHXB sample is in agreement with the ubiquity of low frequency QPOs and hence spin-orbit misalignment in almost all BHXBs.

\section{ACKNOWLEDGEMENTS}

We would like to thank Poshak Gandhi for his useful inputs on the low mass X-ray binary prior to be used for our Bayesian Inference Analysis. PyRAF is a product of the Space Telescope Science Institute, which is operated by AURA for NASA. The Long Baseline Array is part of the Australia Telescope National Facility which is funded by the Australian Government for operation as a National Facility managed by CSIRO. We would like to thank the Institute of Radio Astronomy and Space Research, AUT University, New Zealand for the use and operational support of their radio telescopes in the collection of data for this work. This work made use of the Swinburne University of Technology software correlator, developed as part of the Australian Major National Research Facilities Programme. The Australian SKA Pathfinder is part of the Australia Telescope National Facility which is managed by CSIRO. Operation of ASKAP is funded by the Australian Government with support from the National Collaborative Research Infrastructure Strategy. ASKAP uses the resources of the Pawsey Supercomputing Centre. Establishment of ASKAP, the Murchison Radio-astronomy Observatory and the Pawsey Supercomputing Centre are initiatives of the Australian Government, with support from the Government of Western Australia and the Science and Industry Endowment Fund. We acknowledge the Wajarri Yamatji people as the traditional owners of the Observatory site. This work was supported by resources provided by the Pawsey Supercomputing Centre with funding from the Australian Government and the Government of Western Australia. The European VLBI Network is a joint facility of independent European, African, Asian, and North American radio astronomy institutes. Scientific results from data presented in this publication are derived from the following EVN project code(s): EM101. The Long Baseline Observatory is a facility of the National Science Foundation operated under cooperative agreement by Associated Universities, Inc. The GBT is part of the Green Bank Observatory, a facility of the National Science Foundation operated under a cooperative agreement by Associated Universities, Inc. This work has made use of data from the European Space Agency (ESA) mission Gaia (https: //www.cosmos.esa.int/gaia), processed by the Gaia Data Processing and Analysis Consortium (DPAC,https://www . cosmos.esa.int/web/gaia/dpac/consortium). Funding for the DPAC has been provided by national institutions, in particular the institutions participating in the Gaia Multilateral Agreement. JCAM-J is the recipient of an Australian Research Council Future Fellowship (FT140101082) funded by the Australian government . PGJ acknowledges funding from the European Research Council under ERC Consolidator Grant agreement number 647208. MAPT acknowledge support by the Spanish Ministry of Economy, Industry and Competitiveness (MINECO) under grant AYA2017-83216-P and support via a Ramón y Cajal Fellowship (RYC-201517854). SC acknowledges funding from CNES (french space agency), through MINE, the Multi-wavelength INTEGRAL Network.

\section{REFERENCES}

Abbott B. P., et al., 2016a, Physical Review X, 6, 041015

Abbott B. P., et al., 2016b, ApJ, 818, L22

Adams S. M., Kochanek C. S., Gerke J. R., Stanek K. Z., 2017, MNRAS, 469, 1445

Akaike H., 1974, IEEE Transactions on Automatic Control, 19, 716

Antonini F., Gieles M., Gualandris A., 2018, arXiv e-prints,

Arzoumanian Z., Chernoff D. F., Cordes J. M., 2002, ApJ, 568, 289

Astraatmadja T. L., Bailer-Jones C. A. L., 2016, ApJ, 832, 137

Bahramian A., et al., 2014, ApJ, 780, 127

Bailer-Jones C. A. L., 2015, PASP, 127, 994

Ballet J., Denis M., Gilfanov M., Sunyaev R., Harmon B. A., Zhang S. N., Paciesas W. S., Fishman G. J., 1993, IAU Circ., 5874

Bassi T., et al., 2019, MNRAS, 482, 1587

Bavera S. S., et al., 2019, arXiv e-prints, p. arXiv:1906.12257

Belczynski K., Bulik T., Kalogera V., 2002, ApJ, 571, L147

Belczynski K., Sadowski A., Rasio F. A., Bulik T., 2006, ApJ, 650,303

Belloni T. M., Motta S. E., 2016, in Bambi C., ed., Astrophysics and Space Science Library Vol. 440, Astrophysics of Black Holes: From Fundamental Aspects to Latest Developments. p. 61 (arXiv:1603.07872), doi:10.1007/978-3-662-52859-4_2

Benacquista M. J., Downing J. M. B., 2013, Living Reviews in Relativity, 16, 4

Betancourt M., 2017, arXiv e-prints,

Blaauw A., 1961, Bull. Astron. Inst. Netherlands, 15, 265

Bovy J., 2014, galpy: Galactic dynamics package, Astrophysics Source Code Library (ascl:1411.008)

Brandt W. N., Podsiadlowski P., Sigurdsson S., 1995, MNRAS, 277, L35

Burnham K. P., Anderson D. R., 2002, in Model Selection and Multimodel Inference. , doi:10.1007/b97636

Buxton M., Hasan I., MacPherson E., Bailyn C., 2013, The Astronomer's Telegram, 5244

Cadolle Bel M., et al., 2007, ApJ, 659, 549

Camilo F., Lorimer D. R., Freire P., Lyne A. G., Manchester R. N., 2000, ApJ, 535, 975

Cantrell A. G., et al., 2010, ApJ, 710, 1127

Casares J., 2007, in Karas V., Matt G., eds, IAU Symposium Vol. 238, Black Holes from Stars to Galaxies - Across the Range of Masses. pp 3-12 (arXiv:astro-ph/0612312), doi:10.1017/S1743921307004590

Casares J., Charles P. A., 1994, MNRAS, 271, L5

Casares J., Zurita C., Shahbaz T., Charles P. A., Fender R. P., 2004, ApJ, 613, L133

Casares J., et al., 2009, ApJS, 181, 238

Cavanaugh J. E., 1997, Statistics \& Probability Letters, 33, 201

Chaty S., Mirabel I. F., Goldoni P., Mereghetti S., Duc P.-A., Martí J., Mignani R. P., 2002, MNRAS, 331, 1065 
Chomiuk L., Strader J., Maccarone T. J., Miller-Jones J. C. A., Heinke C., Noyola E., Seth A. C., Ransom S., 2013, ApJ, 777, 69

Chugai N. N., 1984, Soviet Astronomy Letters, 10, 87

Clark G. W., 1975, ApJ, 199, L143

Corral-Santana J. M., Casares J., Muñoz-Darias T., Bauer F. E., Martínez-Pais I. G., Russell D. M., 2016, A\&A, 587, A61

Dehnen W., Binney J., 1998, MNRAS, 294, 429

Deller A. T., et al., 2011, PASP, 123, 275

Dhawan V., Mirabel I. F., Ribó M., Rodrigues I., 2007, ApJ, 668, 430

Drukier G. A., 1996, MNRAS, 280, 498

Fabian A. C., Pringle J. E., Rees M. J., 1975, MNRAS, 172, 15p

Farr W. M., Stevenson S., Miller M. C., Mandel I., Farr B., Vecchio A., 2017, Nature, 548, 426

Fender R. P., Homan J., Belloni T. M., 2009, MNRAS, 396, 1370

Fragos T., McClintock J. E., 2015, ApJ, 800, 17

Fragos T., Willems B., Kalogera V., Ivanova N., Rockefeller G., Fryer C. L., Young P. A., 2009, ApJ, 697, 1057

Fryer C. L., Kalogera V., 2001, ApJ, 554, 548

Fryer C., Burrows A., Benz W., 1998, ApJ, 496, 333

Fuller J., Ma L., 2019, arXiv e-prints, p. arXiv:1907.03714

Gaia Collaboration et al., 2018, A\&A, 616, A1

Gandhi P., Rao A., Johnson M. A. C., Paice J. A., Maccarone T. J., 2019, MNRAS, 485, 2642

García J. A., et al., 2018, ApJ, 864, 25

Gelino D. M., Balman Ş., Kızıloğlu Ü., Yılmaz A., Kalemci E., Tomsick J. A., 2006, ApJ, 642, 438

Gelman A., Rubin D. B., 1992, Statistical Science, 7, 457

Gerosa D., Kesden M., Berti E., O'Shaughnessy R., Sperhake U., 2013, Phys. Rev. D, 87, 104028

Gessner A., Janka H.-T., 2018, ApJ, 865, 61

Gies D. R., et al., 2008, ApJ, 678, 1237

Giesers B., et al., 2018, MNRAS, 475, L15

Giesler M., Clausen D., Ott C. D., 2018, MNRAS, 477, 1853

Gilmore G., Reid N., 1983, MNRAS, 202, 1025

Gnedin O. Y., Zhao H., Pringle J. E., Fall S. M., Livio M., Meylan G., 2002, ApJ, 568, L23

González Hernández J. I., Casares J., 2010, A\&A, 516, A58

González Hernández J. I., Rebolo R., Israelian G., Harlaftis E. T., Filippenko A. V., Chornock R., 2006, ApJ, 644, L49

González Hernández J. I., Rebolo R., Israelian G., Filippenko A. V., Chornock R., Tominaga N., Umeda H., Nomoto K., 2008, ApJ, 679, 732

Goodman J., Hut P., 1993, ApJ, 403, 271

Gourgoulhon E., 1991, A\&A, 252, 651

Gourgoulhon E., Haensel P., 1993, A\&A, 271, 187

Greisen E. W., 2003, in Heck A., ed., Astrophysics and Space Science Library Vol. 285, Information Handling in Astronomy - Historical Vistas. p. 109, doi:10.1007/0-306-48080-8_7

Grimm H.-J., Gilfanov M., Sunyaev R., 2002, A\&A, 391, 923

Gualandris A., Colpi M., Portegies Zwart S., Possenti A., 2005, ApJ, 618, 845

Heggie D. C., Giersz M., 2014, MNRAS, 439, 2459

Heida M., Jonker P. G., Torres M. A. P., Chiavassa A., 2017, ApJ, 846,132

Heise J., 1999, Nuclear Physics B Proceedings Supplements, 69, 186

Hjellming R. M., Rupen M. P., 1995, Nature, 375, 464

Hobbs G., Lorimer D. R., Lyne A. G., Kramer M., 2005, MNRAS, 360,974

Hoffman M. D., Gelman A., 2011, arXiv e-prints,

Hogg D. W., Bovy J., Lang D., 2010, arXiv e-prints,

Homan J., Belloni T., 2005, Ap\&SS, 300, 107

Hynes R. I., 2005, ApJ, 623, 1026

Ingram A., van der Klis M., Middleton M., Done C., Altamirano D., Heil L., Uttley P., Axelsson M., 2016, MNRAS, 461, 1967 Janka H.-T., 2013, MNRAS, 434, 1355
Janka H.-T., 2017, ApJ, 837, 84

Johnson D. R. H., Soderblom D. R., 1987, AJ, 93, 864

Jonker P. G., Nelemans G., 2004, MNRAS, 354, 355

Kalemci E., Dinçer T., Tomsick J. A., Buxton M. M., Bailyn C. D., Chun Y. Y., 2013, ApJ, 779, 95

Karitskaya E. A., Goranskij V. P., 1995, Space Sci. Rev., 74, 489 Kawamuro T., et al., 2018, The Astronomer's Telegram, 11399

Kelley L. Z., Ramirez-Ruiz E., Zemp M., Diemand J., Mandel I., 2010, ApJ, 725, L91

Khargharia J., Froning C. S., Robinson E. L., 2010, ApJ, 716, 1105

Khargharia J., Froning C. S., Robinson E. L., Gelino D. M., 2013, AJ, 145, 21

Khokhlov A. M., Höflich P. A., Oran E. S., Wheeler J. C., Wang L., Chtchelkanova A. Y., 1999, ApJ, 524, L107

Kirsten F., Vlemmings W., Freire P., Kramer M., Rottmann H., Campbell R. M., 2014, A\&A, 565, A43

Kreidberg L., Bailyn C. D., Farr W. M., Kalogera V., 2012, ApJ, 757,36

Kulkarni S. R., Hut P., McMillan S., 1993, Nature, 364, 421

Lindegren L., et al., 2016, A\&A, 595, A4

Luri X., et al., 2018, A\&A, 616, A9

Lyne A. G., Lorimer D. R., 1994, Nature, 369, 127

MacDonald R. K. D., et al., 2014, ApJ, 784, 2

Maccarone T. J., 2003, A\&A, 409, 697

Mandel I., 2010, Phys. Rev. D, 81, 084029

Mandel I., 2016, MNRAS, 456, 578

Markert T. H., Canizares C. R., Clark G. W., Lewin W. H. G., Schnopper H. W., Sprott G. F., 1973, ApJ, 184, L67

Martin R. G., Reis R. C., Pringle J. E., 2008, MNRAS, 391, L15

Masetti N., Bianchini A., Bonibaker J., della Valle M., Vio R., 1996, A\&A, 314, 123

McClintock J. E., Narayan R., Steiner J. F., 2014, Space Sci. Rev., 183,295

Mignard F., 2000, A\&A, 354, 522

Miller-Jones J. C. A., Jonker P. G., Nelemans G., Portegies Zwart S., Dhawan V., Brisken W., Gallo E., Rupen M. P., 2009a, MNRAS, 394, 1440

Miller-Jones J. C. A., Jonker P. G., Dhawan V., Brisken W., Rupen M. P., Nelemans G., Gallo E., 2009b, ApJ, 706, L230

Miller-Jones J. C. A., et al., 2015, MNRAS, 453, 3918

Miller-Jones J. C. A., et al., 2018, MNRAS, 479, 4849

Mirabel F., 2017, New Astron. Rev., 78, 1

Mirabel I. F., Rodrigues I., 2003, Science, 300, 1119

Mirabel I. F., Dhawan V., Mignani R. P., Rodrigues I., Guglielmetti F., 2001, Nature, 413, 139

Mirabel I. F., Mignani R., Rodrigues I., Combi J. A., Rodríguez L. F., Guglielmetti F., 2002, A\&A, 395, 595

Neal R. M., 2012, arXiv e-prints,

Negoro H., et al., 2016, The Astronomer's Telegram, 9876

Nelemans G., Tauris T. M., van den Heuvel E. P. J., 1999, A\&A, $352, \mathrm{~L} 87$

O'Shaughnessy R., Gerosa D., Wysocki D., 2017, Physical Review Letters, 119, 011101

Orosz J. A., Bailyn C. D., McClintock J. E., Remillard R. A., 1996, ApJ, 468, 380

Orosz J. A., Jain R. K., Bailyn C. D., McClintock J. E., Remillard R. A., 1998, ApJ, 499, 375

Orosz J. A., et al., 2001, ApJ, 555, 489

Orosz J. A., McClintock J. E., Aufdenberg J. P., Remillard R. A., Reid M. J., Narayan R., Gou L., 2011, ApJ, 742, 84

Palmer D. M., Barthelmey S. D., Cummings J. R., Gehrels N., Krimm H. A., Markwardt C. B., Sakamoto T., Tueller J., 2005, The Astronomer's Telegram, 546

Podsiadlowski P., Langer N., Poelarends A. J. T., Rappaport S., Heger A., Pfahl E., 2004, ApJ, 612, 1044

Postnov K. A., Yungelson L. R., 2014, Living Reviews in Relativity, 17,3 
Pradel N., Charlot P., Lestrade J.-F., 2006, A\&A, 452, 1099

Reid M. J., McClintock J. E., Narayan R., Gou L., Remillard R. A., Orosz J. A., 2011, ApJ, 742, 83

Reid M. J., McClintock J. E., Steiner J. F., Steeghs D., Remillard R. A., Dhawan V., Narayan R., 2014, ApJ, 796, 2

Repetto S., Davies M. B., Sigurdsson S., 2012, MNRAS, 425, 2799

Repetto S., Igoshev A. P., Nelemans G., 2017, MNRAS, 467, 298

Reynolds T. M., Fraser M., Gilmore G., 2015, MNRAS, 453, 2885

Rodriguez C. L., Haster C.-J., Chatterjee S., Kalogera V., Rasio F. A., 2016, ApJ, 824, L8

Russell T. D., Soria R., Motch C., Pakull M. W., Torres M. A. P., Curran P. A., Jonker P. G., Miller-Jones J. C. A., 2014, MNRAS, 439, 1381

Russell T. D., et al., 2015, MNRAS, 450, 1745

Sagert I., Schaffner-Bielich J., 2008, A\&A, 489, 281

Salvatier J., Wieckiâ T. V., Fonnesbeck C., 2016, PyMC3: Python probabilistic programming framework, Astrophysics Source Code Library (ascl:1610.016)

Shahbaz T., 2003, MNRAS, 339, 1031

Shahbaz T., van der Hooft F., Casares J., Charles P. A., van Paradijs J., 1999, MNRAS, 306, 89

Shahbaz T., Fender R., Charles P. A., 2001, A\&A, 376, L17

Shapiro I. I., et al., 1979, AJ, 84, 1459

Shaw A. W., Charles P. A., Casares J., Hernández Santisteban J. V., 2016, MNRAS, 463, 1314

Sigurdsson S., Hernquist L., 1993, Nature, 364, 423

Sigurdsson S., Phinney E. S., 1993, ApJ, 415, 631

Sippel A. C., Hurley J. R., 2013, MNRAS, 430, L30

Sreehari H., Iyer N., Radhika D., Nandi A., Mandal S., 2018, preprint, (arXiv:1811.04341)

Steeghs D., McClintock J. E., Parsons S. G., Reid M. J., Littlefair S., Dhillon V. S., 2013, ApJ, 768, 185

Stella L., Vietri M., 1998, ApJ, 492, L59

Strader J., Chomiuk L., Maccarone T. J., Miller-Jones J. C. A., Seth A. C., 2012, Nature, 490, 71

Sukhbold T., Ertl T., Woosley S. E., Brown J. M., Janka H.-T., 2016, ApJ, 821, 38

Tauris T. M., Langer N., Podsiadlowski P., 2015, MNRAS, 451, 2123

Tetarenko B. E., Sivakoff G. R., Heinke C. O., Gladstone J. C., 2016a, ApJS, 222, 15

Tetarenko B. E., et al., 2016b, ApJ, 825, 10

The LIGO Scientific Collaboration The Virgo Collaboration 2018, arXiv e-prints,

Verbunt F., Igoshev A., Cator E., 2017, A\&A, 608, A57

White N. E., van Paradijs J., 1996, ApJ, 473, L25

Wijnands R., et al., 2006, A\&A, 449, 1117

Willems B., Henninger M., Levin T., Ivanova N., Kalogera V., McGhee K., Timmes F. X., Fryer C. L., 2005, ApJ, 625, 324

Wong T.-W., Valsecchi F., Fragos T., Kalogera V., 2012, ApJ, 747,111

Woodburn L., Natusch T., Weston S., Thomasson P., Godwin M., Granet C., Gulyaev S., 2015, Publications of the Astronomical Society of Australia, 32, e017

Woosley S. E., Weaver T. A., 1995, ApJS, 101, 181

Wysocki D., Gerosa D., O'Shaughnessy R., Belczynski K., Gladysz W., Berti E., Kesden M., Holz D. E., 2018, Phys. Rev. D, 97, 043014

Xu S., Zhang B., Reid M. J., Zheng X., Wang G., 2019, ApJ, 875, 114

Yan Z., Zhang W., Zhang H., Stiele H., Yu W., 2014, The Astronomer's Telegram, 6649

Ziółkowski J., 2008, Chinese Journal of Astronomy and Astrophysics Supplement, 8, 273

Zurita C., Durant M., Torres M. A. P., Shahbaz T., Casares J., Steeghs D., 2008, ApJ, 681, 1458

della Valle M., Mirabel I. F., Rodriguez L. F., 1994, A\&A, 290, 803 van Grunsven T. F. J., Jonker P. G., Verbunt F. W. M., Robinson

E. L., 2017, MNRAS, 472, 1907

van Paradijs J., White N., 1995, ApJ, 447, L33

This paper has been typeset from a $\mathrm{T}_{\mathrm{E}} \mathrm{X} / \mathrm{LAT} \mathrm{E} \mathrm{X}$ file prepared by the author. 